Pure and Applied Mathematics Quarterly

Volume 7, Number 4

(Special Issue:

In memory of Eckart Viehweg)

1543-1584, 2011

\title{
The Slice Filtration and Grothendieck-Witt Groups
}

\author{
Marc Levine
}

\begin{abstract}
Let $k$ be a perfect field of characteristic different from two. We show that the filtration on the Grothendieck-Witt group GW $(k)$ induced by the slice filtration for the sphere spectrum in the motivic stable homotopy category is the $I$-adic filtration, where $I$ is the augmentation ideal in $\mathrm{GW}(k)$. Keywords: Algebraic cycles, Morel-Voevodsky stable homotopy category, slice filtration.
\end{abstract}

\section{Contents}

Introduction

1. Background and notation

2. The homotopy coniveau tower

3. Connectedness and generators for $\pi_{0}$

4. Cubical generators

5. Transfers and $\mathbb{P}^{1}$-suspension

6. Computing the collapse map

7. Conclusion

Received: Oct. 6, 2010; Revised: Feb. 25, 2011.

2000 Mathematics Subject Classification. Primary 14C25, 19E15; Secondary 19E08 14F42, $55 \mathrm{P} 42$

Research supported by the Alexander von Humboldt Foundation 


\section{INTRODUCTION}

Let $k$ be a perfect field of characteristic different from two. A fundamental theorem of Morel $[8,11]$ states that the endomorphism ring of the motivic sphere spectrum $\mathbb{S}_{k} \in \mathcal{S H}(k)$ is naturally isomorphic to the Grothendieck-Witt ring of quadratic forms over $k, \mathrm{GW}(k)$. This result follows from Morel's calculation [8, corollary 3.43] of the corresponding bi-graded homotopy sheaves of $S^{n} \wedge \mathbb{G}_{m}^{\wedge q}$ in the unstable motivic homotopy category $\mathcal{H}_{\bullet}(k)$ as the Milnor-Witt sheaves (see theorem 7.7 for details), which after stabilizing yields the partial computation of $\pi_{*, *} \mathbb{S}_{k}$ as

$$
\pi_{m+p, p}\left(\mathbb{S}_{k}\right) \cong \begin{cases}\underline{K}_{p}^{M W} & \text { for } m=0 \\ 0 & \text { for } m<0 .\end{cases}
$$

The unstable result also yields the computation of the homotopy sheaf $\pi_{p, p} \Sigma_{s}^{\infty} \mathbb{G}_{m}^{\wedge q}$ (in the $S^{1}$-stable homotopy category $\mathcal{S H}_{S^{1}}(k)$ ) as $\underline{K}_{q-p}^{M W}$, for all $q \geq 1, p \geq 0$.

In another direction, Voevodsky [15] has defined natural towers in $\mathcal{S H}(k)$ and $\mathcal{S H}_{S^{1}}(k)$, which are analogs of the classical Postnikov tower in $\mathcal{S H}$; we call each of these towers the Tate Postnikov tower (in $\mathcal{S H}(k)$ or $\mathcal{S H}_{S^{1}}(k)$, as the case may be). Just as the classical Postnikov tower measures the $S^{n}$-connectivity of a spectrum, the Tate Postnikov tower measures the $S^{*, n}$ connectivity of a motivic spectrum.

In particular, the tower for $\mathbb{S}_{k}$

$$
\ldots \rightarrow f_{n+1} \mathbb{S}_{k} \rightarrow f_{n} \mathbb{S}_{k} \rightarrow \ldots \rightarrow f_{0} \mathbb{S}_{k}=\mathbb{S}_{k}
$$

gives a filtration on the sheaf $\pi_{0,0} \mathbb{S}_{k}$ by

$$
F_{\text {Tate }}^{n} \pi_{0,0} \mathbb{S}_{k}:=\operatorname{im}\left(\pi_{0,0} f_{n} \mathbb{S}_{k} \rightarrow \pi_{0,0} \mathbb{S}_{k}\right)
$$

We have a similarly defined filtration on $\pi_{p, p} \Sigma_{s}^{\infty} \mathbb{G}_{m}^{\wedge q}$, determining $F_{\text {Tate }}^{n} \pi_{0,0} \mathbb{S}_{k}$ by

$$
F_{\text {Tate }}^{n} \pi_{0,0} \mathbb{S}_{k}:=\underset{q}{\lim _{\text {Tate }}} F_{q, q}^{n+q} \Sigma_{s}^{\infty} \mathbb{G}_{m}^{\wedge q}(k) .
$$


Our main result is the computation of $F_{\text {Tate }}^{n} \pi_{p, p} \Sigma_{s}^{\infty} \mathbb{G}_{m}^{\wedge q}$, and thereby $F_{\text {Tate }}^{n} \pi_{0,0} \mathbb{S}_{k}$ (on perfect fields). We first describe the $T$-stable results.

Theorem 1. Let $k$ be a perfect field of characteristic $\neq 2$ and let $F$ be a perfect field extension of $k$. Let $I(F) \subset \mathrm{GW}(F) \cong K_{0}^{M W}(F)$ be the augmentation ideal. 1. Via the identification given by Morel's isomorphism $\pi_{0,0} \mathbb{S}_{k}(F) \cong \mathrm{GW}(F)$, we have

$$
F_{\text {Tate }}^{n} \pi_{0,0} \mathbb{S}_{k}(F)=I(F)^{n}
$$

for all $n \geq 0$. For $n<0, F_{\text {Tate }}^{n} \pi_{0,0} \mathbb{S}_{k}=\pi_{0,0} \mathbb{S}_{k}$ as sheaves.

2. More generally, let $n, p \geq 0, q \geq 1$ be integers. Let $N(a, b)=\max (0, \min (a, b))$. Then via the identification given by Morel's isomorphism $\pi_{p, p} \Sigma_{\mathbb{G}_{m}}^{q} \mathbb{S}_{k} \cong \underline{K}_{q-p}^{M W}$, we have

$$
F_{\text {Tate }}^{n} \pi_{p, p} \Sigma_{\mathbb{G}_{m}}^{q} \mathbb{S}_{k}(F)=K_{q-p}^{M W}(F) I(F)^{N(n-p, n-q)}, n, p, q \in \mathbb{Z}
$$

The stable result follows from the unstable version.

Theorem 2. Let $k$ be a perfect field of characteristic $\neq 2$ and let $F$ be a perfect field extension of $k$. Let $n, p \geq 0, q \geq 1$ be integers. Then via the identification given by Morel's isomorphism $\pi_{p, p} \Sigma_{s}^{\infty} \mathbb{G}_{m}^{\wedge q} \cong \underline{K}_{q-p}^{M W}$, we have

$$
F_{\text {Tate }}^{n} \pi_{p, p} \Sigma_{s}^{\infty} \mathbb{G}_{m}^{\wedge q}(F)=K_{q-p}^{M W}(F) \cdot I(F)^{N(n-p, n-q)} .
$$

See theorem 7.13, corollary 7.14 and corollary 7.15 for details.

Remark 1 . In case $k$ is a field of characteristic 0 , we have a finer result, namely the identities stated in theorem 1 and theorem 2 extend to identities on the corresponding sheaves, for example

$$
F_{\text {Tate }}^{n} \pi_{p, p} \Sigma_{s}^{\infty} \mathbb{G}_{m}^{\wedge q}=\underline{K}_{q-p}^{M W} \cdot \mathcal{I}^{N(n-p, n-q)} .
$$

Of course, one can more generally consider the filtration $F_{\text {Tate }}^{*} \pi_{a, b} \mathcal{E}$ on the homotopy sheaves $\pi_{a, b} \mathcal{E}$ induced by the Tate Postnikov tower for an arbitrary $T$-spectrum $\mathcal{E} \in \mathcal{S H}(k)$. In general, we cannot say anything about this filtration, but assuming a certain connectedness condition, we can compute the filtration on the first non-vanishing homotopy sheaves, evaluated on perfect fields. 
Theorem 3. Let $k$ be a perfect field of characteristic $\neq 2$ and let $F$ be a perfect field extension of $k$. Take $\mathcal{E} \in \mathcal{S H}(k)$ and suppose that $\pi_{a+b, b} \mathcal{E}=0$ for $a<0$, $b \in \mathbb{Z}$. Then for $n>p$,

$$
F_{\text {Tate }}^{n} \pi_{p, p} \mathcal{E}(F)=\left[\pi_{n, n} \mathcal{E} \cdot \underline{K}_{n-p}^{M W}\right]^{T r}(F) .
$$

For $n \leq p$, we have the identity of sheaves

$$
F_{\text {Tate }}^{n} \pi_{p, p} \mathcal{E}=\pi_{p, p} \mathcal{E}
$$

To explain the notation: The canonical action of $\pi_{*, *} \mathbb{S}_{k}$ on $\pi_{*, *} \mathcal{E}$ gives, for each finitely generated field extension $L$ of $k$, a right $K_{-*}^{M W}(L)$-module structure on $\pi_{*, *} \mathcal{E}(L)$, giving us the subgroup $\pi_{n, n} \mathcal{E}(L) \cdot K_{n-p}^{M W}(L)$ of $\pi_{p, p} \mathcal{E}(L)$. This extends to arbitrary field extensions of $k$ by taking the evident colimit. Also, for each closed point $w \in \mathbb{A}_{F}^{n}$, we have a canonically defined transfer map

$$
\operatorname{Tr}_{F}(w)^{*}: \pi_{a, b} \mathcal{E}(F(w)) \rightarrow \pi_{a, b} \mathcal{E}(F)
$$

(see $\S 5$ for details). $\left[\pi_{n, n} \mathcal{E} \cdot \underline{K}_{n-p}^{M W}\right]^{T r}(F)$ is the subgroup of $\pi_{p, p} \mathcal{E}(F)$ generated by the subgroups $\operatorname{Tr}_{F}(w)^{*}\left(\pi_{n, n} \mathcal{E}(F(w)) \cdot K_{n-p}^{M W}(F(w))\right)$, as $w$ runs over closed points of $\mathbb{A}_{F}^{n}$. See theorem 7.11 for details.

Theorem 2 is an easy consequence of theorem 3: one uses Morel's unstable computations of the maps $S^{a, b} \wedge \operatorname{Spec} F_{+} \rightarrow S^{m, n}$ to reduce theorem 2 to its $T$-stable version and then one uses the explicit presentation of $K_{*}^{M W}$ to compute

$$
\left[\underline{K}_{q-n}^{M W} \cdot \underline{K}_{n-p}^{M W}\right]^{T r}(F)=K_{q-p}^{M W}(F) I^{N(n-p, n-q)}(F) .
$$

Morel's results on strictly $\mathbb{A}^{1}$-invariant sheaves allow us to go from the statement on functions fields to the one for the sheaves (in characteristic zero).

The restriction to perfect fields arises from a separability assumption needed to compute the action of transfers on our selected generators for $F_{\text {Tate }}^{n} \pi_{p, p} \mathcal{E}$. We avoid characteristic two so as to have a description of the homotopy sheaves of the sphere spectrum in terms of Milnor-Witt $K$-theory.

The paper is organized as follows. After setting up our notation and going over some background material on motivic homotopy theory in section 1 , we recall some basic facts about the Tate Postnikov tower in section 2. In section 3 we prove some connectedness results for the terms $f_{n} E, s_{n} E$ in the Tate Postnikov tower for an $S^{1}$-spectrum $E$ and give a description of generators for the subgroup $F_{\text {Tate }}^{n} \pi_{0} E(F)$, all under a certain connectedness assumption on $E$. In section 4 , 
we follow a suggestion of the referee and rewrite our generators in cubical form. The generators are then factored into a product of two terms, one depending on $E$, the other only on the choice of a closed point of $\left(\Delta_{F}^{1} \backslash \partial \Delta_{F}^{1}\right)^{n}$. We analyze the second term in sections 6 , having first introduced in section 5 a construction of transfers to allow us to pass from the case of an $F$-point to an arbitrary closed point separable over $F$. In this direction, our main result is a description of this term as a "symbol map" associated to units $u_{1}, \ldots, u_{n} \in F^{\times}$. It is then relatively simple to feed this result into our description of the generators for $F_{\text {Tate }}^{n} \pi_{0} E(F)$ to prove theorems 1 and 3 in section 7 ; we conclude in section 8 with some remarks on the convergence of the Tate Postnikov tower.

I thank the referee for making several helpful suggestions and for pointing out a number of errors, including an incorrect formulation of theorem 3 , in an earlier version of this paper. Finally, I wish to thank the editors for giving me the opportunity of contributing to this volume. As a small token of my gratitude to Eckart for all of his aid and support over many years, I dedicate this article to his memory.

\section{BACKGROUND AND NOTATION}

Unless we specify otherwise, $k$ will be a fixed perfect base field, without restriction on the characteristic. For details on the following constructions, we refer the reader to $[3,4,5,8,9,11,12]$.

We write $[n]:=\{0, \ldots, n\}$ (including $[-1]=\emptyset$ ) and let $\Delta$ be the category with objects $[n], n=0,1, \ldots$, and morphisms $[n] \rightarrow[m]$ the order-preserving maps of sets. Given a category $\mathcal{C}$, the category of simplicial objects in $\mathcal{C}$ is as usual the category of functors $\Delta^{\mathrm{op}} \rightarrow \mathcal{C}$.

Spc will denote the category of simplicial sets, Spc . the category of pointed simplicial sets, $\mathcal{H}:=\mathbf{S p c}\left[W E^{-1}\right]$ the classical unstable homotopy category and $\mathcal{H}_{\bullet}:=\mathbf{S p c}_{\bullet}\left[W E^{-1}\right]$ the pointed version. We denote the suspension operator $-\wedge S^{1}$ by $\Sigma_{s}$. Spt is the category of suspension spectra and $\mathcal{S H}:=\mathbf{S p t}\left[W E^{-1}\right]$ the classical stable homotopy category.

The motivic versions are as follows: $\mathbf{S m} / k$ is the category of smooth finite type $k$-schemes. $\mathbf{S p c}(k)$ is the category of $\mathbf{S p c}$-valued presheaves on $\mathbf{S m} / k, \mathbf{S p c} \bullet(k)$ the $\mathbf{S p c}$-valued presheaves, and $\mathbf{S p t}_{S^{1}}(k)$ the $\mathbf{S p t}$-valued presheaves. These 
all come with "motivic" model structures (see for example [5]); we denote the corresponding homotopy categories by $\mathcal{H}(k), \mathcal{H}_{\bullet}(k)$ and $\mathcal{S H}_{S^{1}}(k)$, respectively. Sending $X \in \mathbf{S m} / k$ to the sheaf of sets on $\mathbf{S m} / k$ represented by $X$ (also denoted $X)$ gives an embedding of $\mathbf{S m} / k$ to $\mathbf{S p c}(k)$; we have the similarly defined embedding of the category of smooth pointed schemes over $k$ into Spc.(k). All these categories are equipped with an internal Hom, denoted $\mathcal{H o m}$.

Let $\mathbb{G}_{m}$ be the pointed $k$-scheme $\left(\mathbb{A}^{1} \backslash 0,1\right)$. In $\mathcal{H}_{\bullet}(k)$ we have the objects $S^{a+b, b}:=\Sigma_{s}^{a} \mathbb{G}_{m}^{\wedge b}$, for $b \geq 1, S^{n, 0}:=S^{n}=\Sigma_{s}^{n} \operatorname{Spec} k_{+}$. If $X$ is a scheme with a $k$-point $x$, we write $(X, x)$ for the corresponding object in $\mathbf{S p c}_{\bullet}(k)$ or $\mathcal{H}_{\bullet}(k)$. For a cofibration $\mathcal{Y} \rightarrow \mathcal{X}$ in $\operatorname{Spc}(k)$, we usually give the quotient $\mathcal{X} / \mathcal{Y}$ the canonical base-point $\mathcal{Y} / \mathcal{Y}$, but on occasion, we will give $\mathcal{X} / \mathcal{Y}$ a base-point coming from a point $x \in \mathcal{X}(k)$; we write this as $(\mathcal{X} / \mathcal{Y}, x)$.

We let $T:=\mathbb{A}^{1} /\left(\mathbb{A}^{1} \backslash\{0\}\right)$ and let $\mathbf{S p t}_{T}(k)$ denote the category of $T$-spectra, i.e., spectra in $\mathbf{S p c}_{\bullet}(k)$ with respect to the $T$-suspension functor $\Sigma_{T}:=-\wedge T$. $\mathbf{S p t}_{T}(k)$ has a motivic model structure (see [5]) and $\mathcal{S H}(k)$ is the homotopy category. We can also form the category of spectra in $\mathbf{S p t}_{S^{1}}(k)$ with respect to $\Sigma_{T}$; with an appropriate model structure the resulting homotopy category is equivalent to $\mathcal{S H}(k)$. We will ignore the subtleties of this distinction and simply identify the two homotopy categories.

Both $\mathcal{S H}_{S^{1}}(k)$ and $\mathcal{S H}(k)$ are triangulated categories with suspension functor $\Sigma_{s}$. We have the triangle of infinite suspension functors $\Sigma^{\infty}$ and their right adjoints $\Omega^{\infty}$
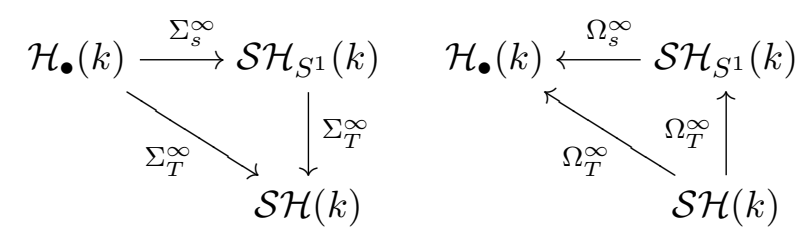

both commutative up to natural isomorphism. These are all left, resp. right derived versions of Quillen adjoint pairs of functors on the underlying model categories. We note that the suspension functor $\Sigma_{\mathbb{G}_{m}}$ is invertible on $\mathcal{S H}(k)$.

For $\mathcal{X} \in \mathcal{H}_{\bullet}(k)$, we have the bi-graded homotopy sheaf $\pi_{a, b} \mathcal{X}$, defined for $b \geq 0$, $a-b \geq 0$, as the Nisnevich sheaf associated to the presheaf on $\mathbf{S m} / k$

$$
U \mapsto \operatorname{Hom}_{\mathcal{H} \bullet}(k)\left(\Sigma_{s}^{a-b} \Sigma_{\mathbb{G}_{m}}^{b} U_{+}, \mathcal{X}\right)
$$


These extend in the usual way to bi-graded homotopy sheaves $\pi_{a, b} E$ for $E \in$ $\mathcal{S H}_{S^{1}}(k), b \geq 0, a \in \mathbb{Z}$, and $\pi_{a, b} \mathcal{E}$ for $\mathcal{E} \in \mathcal{S H}(k), a, b \in \mathbb{Z}$, by taking the Nisnevich sheaf associated to

$$
U \mapsto \operatorname{Hom}_{\mathcal{S H}_{S^{1}}(k)}\left(\Sigma_{s}^{a-b} \Sigma_{\mathbb{G}_{m}}^{b} \Sigma_{s}^{\infty} U_{+}, E\right) \text { or } U \mapsto \operatorname{Hom}_{\mathcal{S H}(k)}\left(\Sigma_{s}^{a-b} \Sigma_{\mathbb{G}_{m}}^{b} \Sigma_{T}^{\infty} U_{+}, \mathcal{E}\right),
$$

as the case may be. We write $\pi_{n}$ for $\pi_{n, 0}$; for e.g. $E \in \mathbf{S p t}_{S^{1}}(k)$ fibrant, $\pi_{n} E$ is the Nisnevich sheaf associated to the presheaf $U \mapsto \pi_{n}(E(U))$.

For $F$ a finitely generated field extension of $k$, we may view $\operatorname{Spec} F$ as the generic point of some $X \in \mathbf{S m} / k$. Thus, for a Nisnevich sheaf $\mathcal{S}$ on $\mathbf{S m} / k$, we may define $\mathcal{S}(F)$ as the stalk of $\mathcal{S}$ at Spec $F \in X$. For an arbitrary field extension $F$ of $k$ (not necessarily finitely generated over $k$ ), we define $\mathcal{S}(F)$ as the colimit over $\mathcal{S}\left(F_{\alpha}\right)$, as $F_{\alpha}$ runs over subfields of $F$ containing $k$ and finitely generated over $k$. For a finitely generated field $F$ over $k$, we consider objects such as $\operatorname{Spec} F$, or $\mathbb{A}_{F}^{n}$ as pro-objects in $\operatorname{Spc}(k)$ by the usual system of finite-type models; the same holds for related objects such as $\operatorname{Spec} F_{+}$in $\mathcal{H}_{\bullet}(k)$ or $\mathcal{S H}_{S^{1}}(k)$, etc. We extend this to arbitrary field extensions of $k$ by taking the system of finitely generated subfields. We will usually not explicitly insert the "pro-" in the text, but all such objects, as well as morphisms and isomorphisms between them, should be so understood.

\section{The homotopy Coniveau tower}

Our computations rely heavily on our model for the Tate Postnikov tower in $\mathcal{S H}_{S^{1}}(k)$, which we briefly recall (for details, we refer the reader to [6]). We start by recalling the Tate Postnikov tower in $\mathcal{S H}_{S^{1}}(k)$ and introducing some notation.

Fix a perfect base-field $k$. Let

$$
\Sigma_{T}: \mathcal{S H}_{S^{1}}(k) \rightarrow \mathcal{S} \mathcal{H}_{S^{1}}(k)
$$

be the $T$-suspension functor. For $n \geq 0$, we let $\Sigma_{T}^{n} \mathcal{S H}_{S^{1}}(k)$ be the localizing subcategory of $\mathcal{S H}_{S^{1}}(k)$ generated by infinite suspension spectra of the form $\Sigma_{T}^{n} \Sigma_{s}^{\infty} X_{+}$, with $X \in \mathbf{S m} / k$. We note that $\Sigma_{T}^{0} \mathcal{S H}_{S^{1}}(k)=\mathcal{S H}_{S^{1}}(k)$. The inclusion functor $i_{n}: \Sigma_{T}^{n} \mathcal{S} \mathcal{H}_{S^{1}}(k) \rightarrow \mathcal{S H}_{S^{1}}(k)$ admits, by results of Neeman [13], a right adjoint $r_{n}$; define the functor $f_{n}: \mathcal{S H}_{S^{1}}(k) \rightarrow \mathcal{S} \mathcal{H}_{S^{1}}(k)$ by $f_{n}:=i_{n} \circ r_{n}$. The unit for the adjunction gives us the natural morphism

$$
\rho_{n}: f_{n} E \rightarrow E
$$


for $E \in \mathcal{S H}_{S^{1}}(k)$; similarly, the inclusion $\Sigma_{T}^{m} \mathcal{S H}_{S^{1}}(k) \subset \Sigma_{T}^{n} \mathcal{S} \mathcal{H}_{S^{1}}(k)$ for $n<m$ gives the natural transformation $f_{m} E \rightarrow f_{n} E$, forming the Tate Postnikov tower

$$
\ldots \rightarrow f_{n+1} E \rightarrow f_{n} E \rightarrow \ldots \rightarrow f_{0} E=E .
$$

We complete $f_{n+1} E \rightarrow f_{n} E$ to a distinguished triangle

$$
f_{n+1} E \rightarrow f_{n} E \rightarrow s_{n} E \rightarrow f_{n+1} E[1] ;
$$

this turns out to be functorial in $E$. The object $s_{n} E$ is the $n$th slice of $E$.

There is an analogous construction in $\mathcal{S H}(k)$ : For $n \in \mathbb{Z}$, let

$$
\Sigma_{T}^{n} \mathcal{S H}^{e f f}(k) \subset \mathcal{S H}(k)
$$

be the localizing category generated by the $T$-suspension spectra $\Sigma_{T}^{n} \Sigma_{T}^{\infty} X_{+}$, for $X \in \mathbf{S m} / k$. As above, the inclusion $i_{n}: \Sigma_{T}^{n} \mathcal{S} \mathcal{H}^{e f f}(k) \rightarrow \mathcal{S H}(k)$ admits a left adjoint $r_{n}$, giving us the truncation functor $f_{n}$ and the Postnikov tower

$$
\ldots \rightarrow f_{n+1} \mathcal{E} \rightarrow f_{n} \mathcal{E} \rightarrow \ldots \rightarrow \mathcal{E} .
$$

Note that this tower is in general infinite in both directions. We define the layer $s_{n} \mathcal{E}$ as above.

By [6, theorem 7.4.1] the 0-space functor $\Omega_{T}^{\infty}$ maps $\Sigma_{T}^{n} \mathcal{S} \mathcal{H}^{e f f}(k)$ to $\Sigma_{T}^{n} \mathcal{S} \mathcal{H}_{S^{1}}(k)$. This fact, together with the universal properties of the truncation functors $f_{n}$ in $\mathcal{S H}_{S^{1}}(k)$ and $\mathcal{S H}(k)$, plus the fact that $\Omega_{T}^{\infty}$ is a right adjoint, gives the canonical isomorphism for $n \geq 0$

$$
f_{n} \Omega_{T}^{\infty} \mathcal{E} \cong \Omega_{T}^{\infty} f_{n} \mathcal{E}
$$

Indeed, it follows from [6, theorem 7.4.1] that $\Omega_{T}^{\infty} f_{n} \mathcal{E}$ is in $\Sigma_{T}^{n} \mathcal{S} \mathcal{H}_{S^{1}}(k)$ and thus we need only show that $\Omega^{\infty} \rho_{n}: \Omega_{T}^{\infty} f_{n} \mathcal{E} \rightarrow \Omega_{T}^{\infty} \mathcal{E}$ satisfies the universal property of $f_{n} \Omega_{T}^{\infty} \mathcal{E} \rightarrow \Omega_{T}^{\infty} \mathcal{E}$. But for $G \in \mathcal{S H}_{S^{1}}(k)$, we have

$$
\begin{aligned}
\operatorname{Hom}_{\mathcal{S H}_{S^{1}}(k)}\left(\Sigma_{T}^{n} G, \Omega_{T}^{\infty} f_{n} \mathcal{E}\right) & \cong \operatorname{Hom}_{\mathcal{S H}(k)}\left(\Sigma_{T}^{\infty} \Sigma_{T}^{n} G, f_{n} \mathcal{E}\right) \\
& \cong \operatorname{Hom}_{\mathcal{S H}(k)}\left(\Sigma_{T}^{n} \Sigma_{T}^{\infty} G, f_{n} \mathcal{E}\right) \\
& \stackrel{\rho_{n *}}{\sim} \operatorname{Hom}_{\mathcal{S H}(k)}\left(\Sigma_{T}^{n} \Sigma_{T}^{\infty} G, \mathcal{E}\right) \\
& \cong \operatorname{Hom}_{\mathcal{S H}(k)}\left(\Sigma_{T}^{\infty} \Sigma_{T}^{n} G, \mathcal{E}\right) \\
& \cong \operatorname{Hom}_{\mathcal{S H}} \mathcal{H}_{S^{1}}(k) \\
& \left(\Sigma_{T}^{n} G, \Omega_{T}^{\infty} \mathcal{E}\right) .
\end{aligned}
$$

It is easy to check that this sequence of isomorphisms is induced by $\left(\Omega_{T}^{\infty} \rho_{n}\right)_{*}$. 
Furthermore, for $E \in \mathcal{S H}_{S^{1}}(k)$, we have (by [6, theorem 7.4.2]) the canonical isomorphism

$$
\Omega_{\mathbb{G}_{m}} f_{n} E=f_{n-1} \Omega_{\mathbb{G}_{m}} E .
$$

As $\Omega_{\mathbb{G}_{m}}: \mathcal{S H}(k) \rightarrow \mathcal{S H}(k)$ is an auto-equivalence, and restricts to an equivalence

$$
\Omega_{\mathbb{G}_{m}}: \Sigma_{T}^{n} \mathcal{S} \mathcal{H}^{e f f}(k) \rightarrow \Sigma_{T}^{n-1} \mathcal{S} \mathcal{H}^{e f f}(k),
$$

the analogous identity in $\mathcal{S H}(k)$ holds as well.

Definition 2.1. For $a \in \mathbb{Z}, b \geq 0, E \in \mathcal{S H}_{S^{1}}(k)$, define the filtration $F_{\text {Tate }}^{n} \pi_{a, b} E$, $n \geq 0$, of $\pi_{a, b} E$ by

$$
F_{\text {Tate }}^{n} \pi_{a, b} E:=\operatorname{im}\left(\pi_{a, b} f_{n} E \rightarrow \pi_{a, b} E\right) .
$$

Similarly, for $\mathcal{E} \in \mathcal{S H}(k), a, b, n \in \mathbb{Z}$, define

$$
F_{\text {Tate }}^{n} \pi_{a, b} \mathcal{E}:=\operatorname{im}\left(\pi_{a, b} f_{n} \mathcal{E} \rightarrow \pi_{a, b} \mathcal{E}\right) .
$$

The main object of this paper is to understand $F_{\text {Tate }}^{n} \pi_{0} E$ for suitable $E$. For later use, we note the following

Lemma 2.2. 1. For $E \in \mathcal{S H}_{S^{1}}(k), n, p, a, b \in \mathbb{Z}$ with $n, p, b, n-p, b-p \geq 0$, the adjunction isomorphism $\pi_{a, b} E \cong \pi_{a-p, b-p} \Omega_{\mathbb{G}_{m}}^{p} E$ induces an isomorphism

$$
F_{\text {Tate }}^{n} \pi_{a, b} E \cong F_{\text {Tate }}^{n-p} \pi_{a-p, b-p} \Omega_{\mathbb{G}_{m}}^{p} E .
$$

Similarly, for $\mathcal{E} \in \mathcal{S H}(k), n, p, a, b \in \mathbb{Z}$, the adjunction isomorphism $\pi_{a, b} \mathcal{E} \cong$ $\pi_{a-p . b-p} \Omega_{\mathbb{G}_{m}}^{p} \mathcal{E}$ induces an isomorphism

$$
F_{\text {Tate }}^{n} \pi_{a, b} \mathcal{E} \cong F_{\text {Tate }}^{n-p} \pi_{a-p, b-p} \Omega_{\mathbb{G}_{m}}^{p} \mathcal{E} .
$$

2. For $\mathcal{E} \in \mathcal{S H}(k), a, b, n \in \mathbb{Z}$, with $b, n \geq 0$, we have a canonical isomorphism

$$
\varphi_{\mathcal{E}, a, b, n}: \pi_{a, b} f_{n} \mathcal{E} \rightarrow \pi_{a, b} f_{n} \Omega_{T}^{\infty} \mathcal{E}
$$

inducing an isomorphism $F_{\text {Tate }}^{n} \pi_{a, b} \mathcal{E} \cong F_{\text {Tate }}^{n} \pi_{a, b} \Omega_{T}^{\infty} \mathcal{E}$.

Proof. (1) By (2.2), adjunction induces isomorphisms

$$
\begin{aligned}
F_{\text {Tate }}^{n} \pi_{a, b} E & :=\operatorname{im}\left(\pi_{a, b} f_{n} E \rightarrow \pi_{a, b} E\right) \\
& \cong \operatorname{im}\left(\pi_{a-p, b-p} \Omega_{\mathbb{G}_{m}}^{p} f_{n} E \rightarrow \pi_{a-p, b-p} \Omega_{\mathbb{G}_{m}}^{p} E\right) \\
& =\operatorname{im}\left(\pi_{a-p, b-p} f_{n-p} \Omega_{\mathbb{G}_{m}}^{p} E \rightarrow \pi_{a-p, b-p} \Omega_{\mathbb{G}_{m}}^{p} E\right) \\
& =F_{\text {Tate }}^{n-p} \pi_{a-p, b-p} \Omega_{\mathbb{G}_{m}}^{p} E .
\end{aligned}
$$


The proof for $\mathcal{E} \in \mathcal{S H}(k)$ is the same.

For (2), the isomorphism $\varphi_{\mathcal{E}, a, b, n}$ arises from (2.1) and the adjunction isomorphism

$$
\begin{aligned}
\operatorname{Hom}_{\mathcal{S H}_{S^{1}}(k)}\left(\Sigma_{s}^{\infty} \Sigma_{s}^{a-b} \Sigma_{\mathbb{G}_{m}}^{b} U_{+}, f_{n} \Omega_{T}^{\infty} \mathcal{E}\right) & \cong \operatorname{Hom}_{\mathcal{S H}} \mathcal{H}_{S^{1}}(k)\left(\Sigma_{s}^{\infty} \Sigma_{s}^{a-b} \Sigma_{\mathbb{G}_{m}}^{b} U_{+}, \Omega_{T}^{\infty} f_{n} \mathcal{E}\right) \\
& \cong \operatorname{Hom}_{\mathcal{S H}(k)}\left(\Sigma_{T}^{\infty} \Sigma_{s}^{a-b} \Sigma_{\mathbb{G}_{m}}^{b} U_{+}, \mathcal{E}\right) .
\end{aligned}
$$

We now turn to a discussion of our model for $f_{n} E(X), X \in \mathbf{S m} / k$. We start with the cosimplicial scheme $n \mapsto \Delta^{n}$, with $\Delta^{n}$ the algebraic $n$-simplex Spec $k\left[t_{0}, \ldots, t_{n}\right] / \sum_{i} t_{i}-1$. The cosimplicial structure is given by sending a map $g:[n] \rightarrow[m]$ to the map $g: \Delta^{n} \rightarrow \Delta^{m}$ determined by

$$
g^{*}\left(t_{i}\right)= \begin{cases}\sum_{j, g(j)=i} t_{j} & \text { if } g^{-1}(i) \neq \emptyset \\ 0 & \text { else. }\end{cases}
$$

A face of $\Delta^{m}$ is a closed subscheme $F$ defined by equations $t_{i_{1}}=\ldots=t_{i_{r}}=0$; we let $\partial \Delta^{n} \subset \Delta^{n}$ be the closed subscheme defined by $\prod_{i=0}^{n} t_{i}=0$, i.e., $\partial \Delta^{n}$ is the union of all the proper faces.

Take $X \in \mathbf{S m} / k$. We let $\mathcal{S}_{X}^{(q)}(m)$ denote the set of closed subsets $W \subset X \times \Delta^{m}$ such that $\operatorname{codim}_{X \times F} W \cap X \times F \geq q$ for all faces $F \subset \Delta^{m}$ (including $F=\Delta^{m}$ ). We make $\mathcal{S}_{X}^{(q)}(m)$ into a partially ordered set via inclusions of closed subsets. Sending $m$ to $\mathcal{S}_{X}^{(q)}(m)$ and $g:[n] \rightarrow[m]$ to $g^{-1}: \mathcal{S}_{X}^{(q)}(m) \rightarrow \mathcal{S}_{X}^{(q)}(n)$ gives us the simplicial poset $\mathcal{S}_{X}^{(q)}$.

Now take $E \in \mathbf{S p t}_{S^{1}}(k)$. For $X \in \mathbf{S m} / k$ and closed subset $W \subset X$, we have the spectrum with supports $E^{W}(X)$ defined as the homotopy fiber of the restriction map $E(X) \rightarrow E(X \backslash W)$. This construction is functorial in the pair $(X, W)$, where we define a map $f:(Y, T) \rightarrow(X, W)$ as a morphism $f: Y \rightarrow X$ in $\mathbf{S m} / k$ with $f^{-1}(W) \subset T$.

Define

$$
E^{(q)}(X, m):=\underset{W \in \mathcal{S}_{X}^{(q)}(m)}{\operatorname{hocolim}} E^{W}\left(X \times \Delta^{m}\right) .
$$

The fact that $m \mapsto \mathcal{S}_{X}^{(q)}(m)$ is a simplicial poset, and $(Y, T) \mapsto E^{T}(Y)$ is a functor from the category of pairs to spectra shows that $m \mapsto E^{(q)}(X, m)$ defines a simplicial spectrum. We denote the associated total spectrum by $E^{(q)}(X)$. 
For $q \geq q^{\prime}$, the inclusions $\mathcal{S}_{X}^{(q)}(m) \subset \mathcal{S}_{X}^{\left(q^{\prime}\right)}(m)$ induces a map of simplicial posets $\mathcal{S}_{X}^{(q)} \subset \mathcal{S}_{X}^{\left(q^{\prime}\right)}$ and thus a morphism of spectra $i_{q^{\prime}, q}: E^{(q)}(X) \rightarrow E^{\left(q^{\prime}\right)}(X)$. We have as well the natural map

$$
\epsilon_{X}: E(X) \rightarrow \operatorname{Tot}\left(E\left(X \times \Delta^{*}\right)\right)=E^{(0)}(X),
$$

which is a weak equivalence if $E$ is homotopy invariant. Together, this forms the augmented homotopy coniveau tower tower

$E^{(*)}(X):=\ldots \rightarrow E^{(q+1)}(X) \stackrel{i_{q}}{\longrightarrow} E^{(q)}(X) \stackrel{i_{q-1}}{\longrightarrow} \ldots E^{(1)}(X) \stackrel{i_{0}}{\longrightarrow} E^{(0)}(X) \stackrel{\epsilon_{X}}{\longleftarrow} E(X)$

with $i_{q}:=i_{q, q+1}$. Thus, for homotopy invariant $E$, we have the homotopy coniveau tower in $\mathcal{S H}$

$$
E^{(*)}(X):=\ldots \rightarrow E^{(q+1)}(X) \stackrel{i_{q}}{\longrightarrow} E^{(q)}(X) \stackrel{i_{q-1}}{\longrightarrow} \ldots E^{(1)}(X) \stackrel{i_{0}}{\longrightarrow} E^{(0)}(X) \cong E(X) .
$$

Letting $\mathbf{S m} / / k$ denote the subcategory of $\mathbf{S m} / k$ with the same objects and with morphisms the smooth morphisms, it is not hard to see that sending $X$ to $E^{(*)}(X)$ defines a functor from $\mathbf{S m} / / k^{\text {op }}$ to augmented towers of spectra.

On the other hand, for $E \in \mathbf{S p t}_{S^{1}}(k)$, we have the (augmented) Tate Postnikov tower

$$
f_{*} E:=\ldots \rightarrow f_{q+1} E \rightarrow f_{q} E \rightarrow \ldots \rightarrow f_{0} E \cong E
$$

in $\mathcal{S H}_{S^{1}}(k)$, which we may evaluate at $X \in \mathbf{S m} / k$, giving the tower $f_{*} E(X)$ in $\mathcal{S H}$, augmented over $E(X)$.

As a direct consequence of our main result (theorem 7.1.1) from [6] we have

Theorem 2.3. Let $E$ be a quasi-fibrant object in $\mathbf{S p t}_{S^{1}}(k)$ for the $\mathbb{A}^{1}$-model structure (see e.g. [5] or [11]), and take $X \in \mathbf{S m} / k$. Then there is an isomorphism of augmented towers in $\mathcal{S H}$

$$
\left(f_{*} E\right)(X) \cong E^{(*)}(X)
$$

over the identity on $E(X)$, which is natural with respect to smooth morphisms in $\mathrm{Sm} / k$.

In particular, we may use the explicit model $E^{(q)}(X)$ to understand $\left(f_{q} E\right)(X)$. Remark 2.4. For $X, Y \in \mathbf{S m} / k$ with given $k$-points $x \in X(k), y \in Y(k)$, we have a natural isomorphism in $\mathcal{S H}_{S^{1}}(k)$

$$
\Sigma_{s}^{\infty}(X \wedge Y) \oplus \Sigma_{s}^{\infty}(X \vee Y) \cong \Sigma_{s}^{\infty}(X \times Y)
$$


i.e. $\Sigma_{s}^{\infty}(X \wedge Y)$ is a canonically defined summand of $\Sigma_{s}^{\infty}(X \times Y)$. In particular for $E$ a quasi-fibrant object of $\mathbf{S p t}_{S^{1}}(k)$, we have a natural isomorphism in $\mathcal{S H}$

$$
\mathcal{H o m}\left(\Sigma_{s}^{\infty}(X \wedge Y), E\right) \cong \operatorname{hofib}(E(X \times Y) \rightarrow \operatorname{hofib}(E(X) \oplus E(Y) \rightarrow E(k)))
$$

where the maps are induced by the evident restriction maps. In particular, we may define $E(X \wedge Y)$ via the above isomorphism, and our comparison results for Tate Postnikov tower and homotopy coniveau tower extend to values at smash products of smooth pointed schemes over $k$.

\section{Connectedness And Generators for $\pi_{0}$}

As in section 2, our base-field $k$ is perfect. We fix $S^{1}$-spectrum $E \in \mathbf{S p t}_{S^{1}}(k)$ that is quasi-fibrant for the $\mathbb{A}^{1}$-model structure, that is, $E$ is $\mathbb{A}^{1}$-homotopy invariant and satisfies Nisnevich excision.

Lemma 3.1. Let $F$ be a finitely generated field extension of $k, x \in \mathbb{A}_{F}^{n}$ a closed point. Then for every $m>0$, the map

$$
i_{0 *}: E^{(x, 0)}\left(\mathbb{A}^{n} \times \mathbb{A}_{F}^{m}\right) \rightarrow E^{\left(x \times_{F} \mathbb{A}_{F}^{m}\right)}\left(\mathbb{A}^{n} \times \mathbb{A}_{F}^{m}\right)
$$

induced by the map of pairs

$$
\operatorname{id}_{\mathbb{A}^{n} \times \mathbb{A}^{m}}:\left(\mathbb{A}^{n} \times \mathbb{A}_{F}^{m}, x \times \mathbb{A}_{F}^{m}\right) \rightarrow\left(\mathbb{A}^{n} \times \mathbb{A}_{F}^{m},(x, 0)\right)
$$

is the zero-map in $\mathcal{S H}$. In particular, the induced map on homotopy groups is the zero map.

Proof. The referee suggested the following simple argument: The map $i_{0 *}$ is induced by applying the functor $H(-):=\operatorname{Hom}_{\mathcal{H} \mathbf{S p t}_{S^{1}}(k)}\left(\Sigma_{s}^{\infty}(-), E\right)$ to the quotient map

$$
q: \mathbb{A}_{F}^{n} \times \mathbb{A}^{m} /\left(\mathbb{A}_{F}^{n} \times \mathbb{A}^{m} \backslash x \times \mathbb{A}^{m}\right) \rightarrow \mathbb{A}_{F}^{n} \times \mathbb{A}^{m} /\left(\mathbb{A}_{F}^{n} \times \mathbb{A}^{m} \backslash\{(x, 0\}),\right.
$$

where the homotopy category $\mathcal{H} \mathbf{S p t}_{S^{1}}(k)$ is with respect to the presheaf model structure on $\mathbf{S p t}_{S^{1}}(k)$, i.e., weak equivalences are objectwise. But $E$ is homotopy invariant, and hence the inclusion

$$
i_{1}:\left(\mathbb{A}_{F}^{n} \times 1, x \times 1\right) \rightarrow\left(\mathbb{A}_{F}^{n} \times \mathbb{A}^{m}, x \times \mathbb{A}^{m}\right)
$$

induces an isomorphism

$$
i_{1}^{*}: H\left(\mathbb{A}_{F}^{n} \times \mathbb{A}^{m} /\left(\mathbb{A}_{F}^{n} \times \mathbb{A}^{m} \backslash x \times \mathbb{A}^{m}\right)\right) \rightarrow H\left(\mathbb{A}_{F}^{n} /\left(\mathbb{A}_{F}^{n} \times 1 \backslash x \times 1\right)\right.
$$


But clearly $q \circ i_{1}$ factors through the map to the base-point, hence $q^{*}$ is the 0 -map on $H$.

We have the re-indexed homotopy sheaves $\Pi_{n, m}(E):=\pi_{n+m, m}(E)$. We have as well the sheaf $\pi_{n} E:=\pi_{n, 0} E$; we call $E m$-connected if $\pi_{n}(E)=0$ for all $n \leq m$.

Since $E^{(n)}(X)=\operatorname{Tot}\left[m \mapsto E^{(n)}(X, m)\right]$, we have the strongly convergent spectral sequence

$$
E_{p, q}^{1}(X)=\pi_{q} E^{(n)}(X, p) \Longrightarrow \pi_{p+q} E^{(n)}(X),
$$

Now take $X=\operatorname{Spec} F, F$ a finitely generated field over $k$. For dimensional reasons, we have $\mathcal{S}_{F}^{(n)}(p)=\emptyset$ for $p<n$, and we therefore have an edge homomorphism

$$
\epsilon_{-n}: \pi_{q-n} E^{(n)}(X, n) \rightarrow \pi_{q} E^{(n)}(X) .
$$

Furthermore, $\mathcal{S}_{F}^{(n)}(n)$ is the set of closed points $w \in \Delta_{F}^{n} \backslash \partial \Delta_{F}^{n}$, so $\epsilon_{-n}$ can be written as

$$
\epsilon_{-n}: \oplus_{w \in\left(\Delta_{F}^{n} \backslash \partial \Delta_{F}^{n}\right)(n)} \pi_{q-n} E^{w}\left(\Delta_{F}^{n}\right) \rightarrow \pi_{q} E^{(n)}(F) ;
$$

here $Y^{(a)}$ denotes the set of codimension $a$ points on a scheme $Y$.

Via the weak equivalence $E^{(n)}(F) \cong f_{n} E(F)$, we have the canonical map

$$
\epsilon_{-n}: \oplus_{w \in\left(\Delta_{F}^{n} \backslash \partial \Delta_{F}^{n}\right)^{(n)}} \pi_{q-n} E^{w}\left(\Delta_{F}^{n}\right) \rightarrow \pi_{q} f_{n} E(F) .
$$

Similarly, composing with $f_{n} E \rightarrow s_{n} E$, we have the canonical map

$$
\epsilon_{-n}: \oplus_{w \in\left(\Delta_{F}^{n} \backslash \partial \Delta_{F}^{n}\right)(n)} \pi_{q-n} E^{w}\left(\Delta_{F}^{n}\right) \rightarrow \pi_{q} s_{n} E(F) .
$$

Proposition 3.2. Let $E \in \mathbf{S p t}_{S^{1}}(k)$ be quasi-fibrant for the $\mathbb{A}^{1}$-model structure. Suppose $\Pi_{a, *} E(F)=0$ for all $a<0$ and for all finitely generated field extensions $F$ of $k$. Then for $n \geq 0$ :

1. $\Pi_{a, *} f_{n} E$ and $\Pi_{a, *} s_{n} E$ are zero for all $a<0$. In particular, $f_{n} E$ and $s_{n} E$ are-1-connected.

2. For each finitely generated field $F$ over $k$, the edge homomorphisms

$$
\begin{aligned}
& \epsilon_{-n}: \oplus_{w \in\left(\Delta_{F}^{n} \backslash \partial \Delta_{F}^{n}\right)^{(n)}} \pi_{-n} E^{w}\left(\Delta_{F}^{n}\right) \rightarrow \pi_{0}\left(f_{n} E\right)(F) \\
& \epsilon_{-n}: \oplus_{w \in\left(\Delta_{F}^{n} \backslash \partial \Delta_{F}^{n}\right)^{(n)}} \pi_{-n} E^{w}\left(\Delta_{F}^{n}\right) \rightarrow \pi_{0}\left(s_{n} E\right)(F)
\end{aligned}
$$

are surjections. 
Proof. Using the distinguished triangle

$$
f_{n+1} E \rightarrow f_{n} E \rightarrow s_{n} E \rightarrow \Sigma_{s} f_{n+1} E
$$

we see that it suffices to prove the statements for $f_{n} E$.

Using the isomorphism (2.2), we see that for (1), it suffices to show that $f_{n} E$ is -1-connected. By a theorem of Morel [11, lemma 3.3.6], it suffices to show that $f_{n} E(F)$ is -1-connected for all finitely generated field extensions $F$ of $k$.

We first show that, for each $p \geq n$,

a. $\pi_{q} E^{(n)}(F, p)=0$ for $q<-p$

b. The natural map

$$
\oplus_{W \in \mathcal{S}_{F}^{(n)}(p), w \in W \cap\left(\Delta_{F}^{p}\right)^{(p)}} \pi_{-p} E^{w}\left(\Delta_{F}^{p}\right) \rightarrow \pi_{-p} E^{(n)}(F, p)
$$

is surjective.

For (a), let $W \subset \Delta_{F}^{p}$ be a closed subset. We have the Gersten spectral sequence

$$
E_{1}^{a, b}=\oplus_{w \in W \cap\left(\Delta_{F}^{p}\right)^{(a)}} \pi_{-a-b} E^{w}\left(\operatorname{Spec} \mathcal{O}_{\Delta_{F}^{p}, w}\right) \Longrightarrow \pi_{-a-b} E^{W}\left(\Delta_{F}^{p}\right) .
$$

Since $E$ is quasi-fibrant, and $\Delta_{F}^{p}$ is smooth over $k$, we have an isomorphism (via Morel-Voevodsky purity [12, Theorem 3.2.23])

$$
\pi_{m}\left(E^{w}\left(\operatorname{Spec} \mathcal{O}_{\Delta_{F}^{p}, w}\right)\right) \cong \pi_{m}\left(E\left(w_{+} \wedge S^{2 a, a}\right)\right),
$$

where $a=\operatorname{codim}_{\Delta_{F}^{p}} w$. But

$$
\pi_{m}\left(E\left(w_{+} \wedge S^{2 a, a}\right)\right)=\left(\pi_{m+2 a, a} E\right)(F(w))
$$

which is zero for $m+a<0$. Since $0 \leq a \leq p$, we see that, for $m<-p$,

$$
\pi_{m} E^{W}\left(\Delta_{F}^{p}\right)=0 \text {. }
$$

As $E^{(n)}(F, p)$ is a colimit over $E^{W}\left(\Delta_{F}^{p}\right)$ with $W \in \mathcal{S}_{F}^{(n)}(p)$, it follows that $\pi_{m} E^{(n)}(F, p)=0$ for $m<-p$, proving (a).

The same computation shows that $\pi_{-p}\left(E^{w}\left(\operatorname{Spec} \mathcal{O}_{\Delta_{F}^{p}, w}\right)\right)=0$ if $\operatorname{codim}_{\Delta_{F}^{p}} w<$ $p$, so (b) follows from the Gersten spectral sequence.

Using the strongly convergent spectral sequence (3.1), we see that (a) implies that $\pi_{q} E^{(n)}(F)=0$ for $q<0$. 
Next, we show that

$$
\text { c. } \pi_{-p} E^{(n)}(F, p)=0 \text { for } p>n \text {. }
$$

For this, it suffices by (b) to show that for $w \in W \cap\left(\Delta_{F}^{p}\right)^{(p)}$ with $W \in \mathcal{S}_{F}^{(n)}(p)$ and with $p>n$, the map

$$
\pi_{-p} E^{w}\left(\Delta_{F}^{p}\right) \rightarrow \pi_{-p} E^{(n)}(F, p)
$$

is the zero map. To see this, note that $W$ does not intersect any face $T$ of $\Delta_{F}^{p}$ having $\operatorname{dim}_{F} T<n$. Thus, there is a linear $W^{\prime} \cong \mathbb{A}_{F^{\prime}}^{p-n} \subset \Delta_{F}^{p}$ containing $w$ (for $F^{\prime}$ some extension field of $F$ contained in $F(w)$ ) with $W^{\prime} \in \mathcal{S}_{F}^{(n)}(p)$ : for a suitable degeneracy map $\sigma: \Delta^{p} \rightarrow \Delta^{n}$ one takes $W^{\prime}=\sigma^{-1}(\sigma(w))$. By lemma 3.1, the map $E^{w}\left(\Delta_{F}^{p}\right) \rightarrow E^{W^{\prime}}\left(\Delta_{F}^{p}\right)$ is the zero map in $\mathcal{S H}$; passing to the limit over all $W^{\prime \prime} \in \mathcal{S}_{F}^{(n)}(p)$, we see that (3.2) is the zero map, as claimed.

In the spectral sequence (3.1), we have $E_{p,-p}^{1}=0$ for $p>n$; we also have $E_{p,-p}^{1}=0$ for $p<n$ since $\mathcal{S}_{F}^{(n)}(p)=\emptyset$ if $p<n$ for dimensional reasons. Thus, the only term contributing to $\pi_{0} E^{(n)}(F)$ is $E_{n,-n}^{1}$. As the spectral sequence is strongly convergent, the edge homomorphism in the spectral sequence (3.1) induces a surjection

$$
\oplus_{w \in \mathcal{S}_{F}^{(n)}(n)} \pi_{-n} E^{w}\left(\Delta_{F}^{n}\right) \rightarrow \pi_{0} E^{(n)}(F) .
$$

Combining this with theorem 2.3 gives us the surjection

$$
\oplus_{w \in \mathcal{S}_{F}^{(n)}(n)} \pi_{-n} E^{w}\left(\Delta_{F}^{n}\right) \rightarrow \pi_{0}\left(f_{n} E(F)\right) .
$$

Similarly, the vanishing $\pi_{p} E^{(n)}(F)=0$ for $p<0$ shows that $f_{n} E(F)$ is -1 connected.

We thus have generators $\oplus_{w \in\left(\Delta_{F}^{n} \backslash \partial \Delta_{F}^{n}\right)(n)} \pi_{-n} E^{w}\left(\Delta_{F}^{n}\right)$ for $\pi_{0} f_{n} E(F)$, and hence for our main object of study, $F_{\text {Tate }}^{n} \pi_{0} E(F)$.

\section{Cubical generators}

As pointed out by the referee, it is much more convenient to work with a cubical type of generator for $F_{\text {Tate }}^{n} \pi_{0} E(F)$. We first look at the generators for $F_{\text {Tate }}^{1} \pi_{0} E(F)$, to put these in a simpler form, which we then extend to give the cubical generators for $F_{\text {Tate }}^{n} \pi_{0} E(F)$ by a simple induction. 
For $F$ a finitely generated field extension of $k$, the contractibility of $\Delta^{1}$ gives us the canonical isomorphism in $\mathcal{H}_{\bullet}(k)$

$$
\sigma_{F}^{1}: \Sigma_{s} \operatorname{Spec} F_{+} \rightarrow \Delta_{F}^{1} / \partial \Delta^{1} .
$$

Let $w=\left(w_{0}, w_{1}\right)$ be an $F$-rational point of $\Delta^{1} \backslash \partial \Delta^{1}$. Writing

$$
\Delta^{1}:=\operatorname{Spec} k\left[t_{0}, t_{1}\right] /\left(t_{0}+t_{1}-1\right),
$$

we map $\Delta_{F}^{1}$ to $\mathbb{A}_{F}^{1}$ by the function

$$
X_{w}:=\frac{t_{1}-w_{1}}{w_{0}}: \Delta_{F}^{1} \rightarrow \mathbb{A}_{F}^{1} .
$$

Clearly

$$
X_{w}(1,0)=-\frac{w_{1}}{w_{0}}, X_{w}(0,1)=1, X_{w}(w)=0 .
$$

Thus $X_{w}$ induced a map of diagrams in $\mathbf{S p c}_{\bullet}(k)$

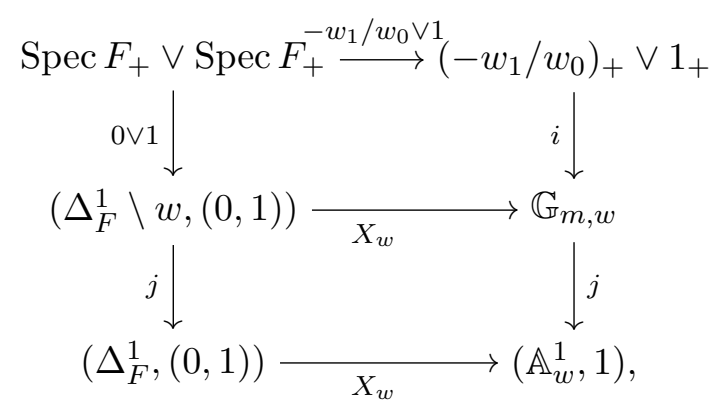

giving us the induced map on the quotients

$$
\Delta_{F}^{1} /\{(0,1),(1,0)\} \stackrel{\pi_{w}}{\longrightarrow} \Delta_{F}^{1} / \Delta_{F}^{1} \backslash\{w\} \stackrel{X_{w}}{\longrightarrow} \mathbb{A}_{w}^{1} / \mathbb{A}_{w}^{1} \backslash\left\{0_{w}\right\} .
$$

In $\mathcal{H}_{\bullet}(k)$, this gives us the sequence of maps

$$
\Sigma_{s} \operatorname{Spec} F_{+} \stackrel{\sigma_{F}^{1}}{\longrightarrow} \Delta_{F}^{1} / \partial \Delta_{F}^{1} \stackrel{\pi_{w}}{\longrightarrow} \Delta_{F}^{1} / \Delta_{F}^{1} \backslash\{w\} \stackrel{X_{w}}{\longrightarrow} \Sigma_{s} w_{+} \wedge \mathbb{G}_{m}
$$

with $X_{w}$ and $\sigma_{F}^{1}$ isomorphisms.

In case $w$ is separable over $F$, let

$$
p_{w}: \Delta_{F(w)}^{1} \rightarrow \Delta_{F}^{1}
$$

be the projection, and let $w \in \Delta_{F(w)}^{1}$ be the point given the the maps $w \rightarrow \Delta_{F}^{1}$, $w=\operatorname{Spec} F(w)$. By Nisnevich excision, $p_{w}$ induces an isomorphism

$$
p_{w}: \Delta_{F(w)}^{1} / \Delta_{F(w)}^{1} \backslash\{w\} \rightarrow \Delta_{F}^{1} / \Delta_{F}^{1} \backslash\{w\},
$$


giving us the sequence of maps in $\mathcal{H}_{\bullet}(k)$, this gives us the sequence of maps

$$
\Sigma_{s} \operatorname{Spec} F_{+} \stackrel{\pi_{w}}{\longrightarrow} \Delta_{F}^{1} / \Delta_{F}^{1} \backslash\{w\} \stackrel{p_{w}^{-1}}{\longrightarrow} \Delta_{F(w)}^{1} / \Delta_{F(w)}^{1} \backslash\{w\} \stackrel{X_{w}}{\longrightarrow} \Sigma_{s} w_{+} \wedge \mathbb{G}_{m}
$$

with $X_{w}$ an isomorphism. We let

$$
M V_{w}: \Delta_{F}^{1} / \Delta_{F}^{1} \backslash\{w\} \rightarrow \Sigma_{s} w_{+} \wedge \mathbb{G}_{m}
$$

denote the isomorphism $X_{w} \circ p_{w}^{-1}$, and we write

$$
(w): \operatorname{Spec} F_{+} \rightarrow w_{+} \wedge \mathbb{G}_{m}
$$

for the map (in $\left.\mathcal{S} \mathcal{H}_{S^{1}}\right) \Sigma^{-1}\left(M V_{w} \circ \pi_{w} \circ \sigma_{F}^{1}\right)$.

For $\alpha \in \pi_{0}\left(\left(\Omega_{\mathbb{G}_{m}} E\right)(w)\right)$, we let $\alpha^{\prime}: w_{+} \wedge \mathbb{G}_{m} \rightarrow E$ denote the corresponding morphism in $\mathcal{S} \mathcal{H}_{S^{1}}(k)$. Noting that $\Omega_{\mathbb{G}_{m}} f_{1} E \cong f_{0} \Omega_{\mathbb{G}_{m}} E \cong \Omega_{\mathbb{G}_{m}} E$, we let $\alpha^{\prime \prime}$ : $w_{+} \wedge \mathbb{G}_{m} \rightarrow f_{1} E$ denote the morphism corresponding to $\alpha^{\prime}$.

Lemma 4.1. Let $E \in \mathbf{S p t}_{S^{1}}(k)$ be quasi-fibrant. Suppose $\Pi_{a, *} E(F)=0$ for all $a<0$ and for all finitely generated field extensions $F$ of $k$. Let $F$ be the perfect closure of a finitely generated field $F_{0}$ over $k$. Then sending $\alpha \in \pi_{0}\left(\left(\Omega_{\mathbb{G}_{m}} f_{1} E\right)(w)\right)$ to the composition $\alpha^{\prime \prime} \circ(w)$ :

$$
\operatorname{Spec} F_{+} \stackrel{\Sigma^{-1} \pi_{w}}{\longrightarrow} \Sigma^{-1} \Delta_{F}^{1} / \Delta_{F}^{1} \backslash\{w\} \stackrel{\Sigma^{-1} M V_{w}}{\longrightarrow} w_{+} \wedge \mathbb{G}_{m} \stackrel{\alpha^{\prime \prime}}{\longrightarrow} f_{1} E(w)
$$

determines a surjection

$$
\tilde{\epsilon}_{-1}: \oplus_{w \in\left(\Delta_{F}^{1} \backslash \partial \Delta_{F}^{1}\right)^{(1)}} \pi_{0}\left(\left(\Omega_{\mathbb{G}_{m}} f_{1} E\right)(w)\right) \rightarrow \pi_{0}\left(f_{1} E(F)\right) .
$$

Proof. The edge homomorphism $\epsilon_{-1}$ of proposition 3.2 sends $\beta \in \pi_{-1}\left(E^{w}\left(\Delta_{F}^{1}\right)\right.$ to the composition

$$
\Sigma \operatorname{Spec} F_{+} \cong \Delta_{F}^{1} /\{(0,1),(1,0)\} \stackrel{\pi_{w}}{\longrightarrow} \Delta_{F}^{1} / \Delta_{F}^{1} \backslash\{w\} \stackrel{\beta^{\prime}}{\longrightarrow} \Sigma E
$$

where $\beta^{\prime}$ is the morphism given by the identity

$$
\pi_{-1}\left(E^{w}\left(\Delta_{F}^{1}\right) \cong \operatorname{Hom}_{\mathcal{S H}_{S^{1}}(k)}\left(\Delta_{F}^{1} / \Delta_{F}^{1} \backslash\{w\}, \Sigma E\right) .\right.
$$

Applying $\Sigma^{-1}$ and inserting id $=M V_{w}^{-1} \circ M V_{w}$, we may rewrite the above composition as

$$
\begin{aligned}
\operatorname{Spec} F_{+} \cong \Sigma^{-1} \Delta_{F}^{1} /\{(0,1),(1,0)\} \stackrel{\Sigma^{-1} \pi_{w}}{\longrightarrow} \Sigma^{-1} \Delta_{F}^{1} / \Delta_{F}^{1} \backslash\{w\} \\
\stackrel{M V_{w}}{\longrightarrow} w_{+} \wedge \mathbb{G}_{m} \stackrel{\Sigma^{-1} \beta^{\prime} \circ M V_{w}^{-1}}{\longrightarrow} E
\end{aligned}
$$


By the universal property of $f_{1} E \rightarrow E$, we may factor the map $\Sigma^{-1} \beta^{\prime} \circ M V_{w}^{-1}$ uniquely as

$$
w_{+} \wedge \mathbb{G}_{m} \stackrel{\alpha^{\prime}}{\longrightarrow} f_{1} E \rightarrow E
$$

giving us the corresponding element $\alpha \in \pi_{0}\left(\left(\Omega_{\mathbb{G}_{m}} f_{1} E\right)(w)\right)$. It is easy to check that for $\alpha \in \pi_{0}\left(\left(\Omega_{\mathbb{G}_{m}} f_{1} E\right)(w)\right)$ corresponding to $\beta \in \pi_{-1}\left(E^{w}\left(\Delta_{F}^{1}\right)\right.$, we have

$$
\epsilon_{-1}(\beta)=\tilde{\epsilon}_{-1}(\alpha)
$$

Clearly we may reverse this process.

We extend this description of the generators for $\pi_{0}\left(f_{1} E\right)$ to $\pi_{0}\left(f_{n} E\right)$ be a simple induction. Let $U \subset X$ and $V \subset Y$ be open subschemes of smooth schemes $X, Y$ over $k$. We have a canonical isomorphism in $\mathcal{H}_{\bullet}^{\mathrm{Nis}}(k)$,

$$
\alpha_{X, Y ; U, V}: X \times_{k} Y / U \times Y \cup X \times V \rightarrow(X / U) \wedge(Y / V),
$$

induced by the Mayer-Vietoris isomorphism

$$
U \times Y \cup X \times V \cong U \times Y \amalg_{U \times V} X \times V .
$$

Applying this to an $F$-point $w=\left(w^{1}, \ldots, w^{n}\right) \in\left(\Delta_{F}^{1} \backslash \partial \Delta_{F}^{1}\right)^{n}$ gives us the isomorphism

$$
\begin{aligned}
\left(\Delta_{F}^{1} \backslash \partial \Delta_{F}^{1}\right)^{n} /\left(\left(\Delta_{F}^{1} \backslash \partial \Delta_{F}^{1}\right)^{n} \backslash\{w\}\right) & \\
& \stackrel{X_{w}:=\left(X_{w^{1}}, \ldots, X_{w^{n}}\right)}{\longrightarrow} \\
& \operatorname{Spec} F_{+} \wedge\left(\Sigma \mathbb{G}_{m}\right)^{\wedge n} \cong \Sigma^{n} \operatorname{Spec} F_{+} \wedge \mathbb{G}_{m}^{\wedge n} .
\end{aligned}
$$

As in the case $n=1$, for a closed point $w$ of $\left(\left(\Delta_{F}^{1} \backslash \partial \Delta_{F}^{1}\right)^{n}\right.$, separable over $F$, we have the Nisnevich excision isomorphism

$$
\begin{aligned}
p_{w}:\left(\Delta_{F(w)}^{1} \backslash \partial \Delta_{F(w)}^{1}\right)^{n} /\left(\left(\Delta_{F(w)}^{1} \backslash\right.\right. & \left.\left.\partial \Delta_{F(w)}^{1}\right)^{n} \backslash\{w\}\right) \\
& \rightarrow\left(\Delta_{F}^{1} \backslash \partial \Delta_{F}^{1}\right)^{n} /\left(\left(\Delta_{F}^{1} \backslash \partial \Delta_{F}^{1}\right)^{n} \backslash\{w\}\right),
\end{aligned}
$$

giving us the isomorphism in $\mathcal{H}_{\bullet}(k)$

$$
\begin{gathered}
M V_{w}:\left(\Delta_{F}^{1} \backslash \partial \Delta_{F}^{1}\right)^{n} /\left(\left(\Delta_{F}^{1} \backslash \partial \Delta_{F}^{1}\right)^{n} \backslash\{w\}\right) \rightarrow w_{+} \wedge \Sigma^{n} \mathbb{G}_{m}^{\wedge n} \\
M V_{w}:=X_{w} \circ p_{w}^{-1} .
\end{gathered}
$$


This gives us the sequence of morphisms in $\mathcal{H}_{\bullet}(k)$

$$
\begin{aligned}
\Sigma_{s}^{n} \operatorname{Spec} F_{+} \stackrel{\sigma_{F}^{n}}{\sim} \operatorname{Spec} F_{+} & \wedge\left(\Delta^{1} / \partial \Delta^{1}\right)^{\wedge n} \\
& \stackrel{\pi_{w}}{\longrightarrow}\left(\Delta_{F}^{1}\right)^{n} /\left(\left(\Delta_{F}^{1}\right)^{n} \backslash\{w\}\right) \stackrel{M V_{w}}{\sim} w_{+} \wedge \Sigma^{n} \mathbb{G}_{m}^{\wedge n}
\end{aligned}
$$

with $\sigma_{F}^{n}$ and $M V_{w}$ isomorphisms and $\pi_{w}$ the quotient map.

As above, we let

$$
(w): \operatorname{Spec} F_{+} \rightarrow w_{+} \wedge \mathbb{G}_{m}^{\wedge n}
$$

be the shifted composition $\Sigma^{-n}\left(M V_{w} \circ \pi_{w} \circ \sigma_{F}^{n}\right)$ in $\mathcal{S H}_{S^{1}}(k)$.

For $\alpha \in \pi_{0}\left(\left(\Omega_{\mathbb{G}_{m}}^{n} E\right)(w)\right)$, we let $\alpha^{\prime}: w_{+} \wedge \mathbb{G}_{m}^{\wedge n} \rightarrow E$ denote the corresponding morphism in $\mathcal{S H}_{S^{1}}(k)$. We note that

$$
\Omega_{\mathbb{G}_{m}}^{n} f_{n} E \cong f_{0} \Omega_{\mathbb{G}_{m}}^{n} E=\Omega_{\mathbb{G}_{m}}^{n} E ;
$$

for $\alpha \in \pi_{0}\left(\left(\Omega_{\mathbb{G}_{m}}^{n} E\right)(w)\right)$ we write $\alpha^{\prime \prime}: w_{+} \wedge \mathbb{G}_{m}^{\wedge n} \rightarrow f_{n} E$ for the morphism corresponding to $\alpha^{\prime}: w_{+} \wedge \mathbb{G}_{m}^{\wedge n} \rightarrow E$.

Proposition 4.2. Let $E \in \mathbf{S p t}_{S^{1}}(k)$ be quasi-fibrant. Suppose $\Pi_{a, *} E(F)=0$ for all $a<0$ and for all finitely generated field extensions $F$ of $k$. Let $F$ be the perfect closure of a finitely generated field $F_{0}$ over $k$. Then sending $\alpha \in \pi_{0}\left(\left(\Omega_{\mathbb{G}_{m}}^{n} E\right)(w)\right)$, $w$ a closed point of $\left(\Delta_{F}^{1} \backslash \partial \Delta_{F}^{1}\right)^{n}$, to the composition $\alpha^{\prime \prime} \circ(w)$,

$$
\operatorname{Spec} F_{+} \stackrel{\Sigma^{-n} \pi_{w} \circ \sigma_{F}^{n}}{\longrightarrow} \Sigma^{-n} \Delta_{F}^{1} /\left(\left(\Delta_{F}^{1}\right)^{n} \backslash\{w\}\right) \stackrel{\Sigma^{-n} M V_{w}}{\longrightarrow} w_{+} \wedge \mathbb{G}_{m}^{\wedge n} \stackrel{\alpha^{\prime \prime}}{\longrightarrow} f_{n} E(w)
$$

determines a surjection

$$
\tilde{\epsilon}_{-n}: \oplus_{\left.w \in\left(\Delta_{F}^{1} \backslash \partial \Delta_{F}^{1}\right)^{n}\right)^{(n)}} \pi_{0}\left(\left(\Omega_{\mathbb{G}_{m}}^{n} E\right)(w)\right) \rightarrow \pi_{0}\left(f_{n} E(F)\right) .
$$

Proof. We proceed by induction on $n$, the case $n=1$ being lemma 4.1. So, take $n>1$ and assume the result is true for $n=1$ and for $n-1$. Since $f_{1} f_{n} \cong f_{n}$, we have generators for $\pi_{0}\left(f_{n} E\right)(F)$ of the form $\tilde{\epsilon}_{-1}(\alpha)$, with $\alpha \in$ $\pi_{0}\left(\left(\Omega_{\mathbb{G}_{m}} f_{1}\left(f_{n} E\right)\right)\left(w^{n}\right)\right)$, and $w^{n}$ a closed point of $\Delta_{F}^{1} \backslash \partial \Delta_{F}^{1}$. But we have the isomorphism

$$
\sigma: \Omega_{\mathbb{G}_{m}} f_{1}\left(f_{n} E\right) \rightarrow \Omega_{\mathbb{G}_{m}} f_{n} E \rightarrow f_{n-1} \Omega_{\mathbb{G}_{m}} E
$$

so by induction we can write $\sigma \circ \alpha$ as a sum of elements of the form $\tilde{\epsilon}_{-(n-1)}(\beta)$, with $\beta \in \pi_{0}\left(\left(\Omega_{\mathbb{G}_{m}}^{n-1} E\right)\left(\left(w^{1}, \ldots, w^{n-1}\right)\right),\left(w^{1}, \ldots, w^{n-1}\right)\right.$ a closed point of $\left(\Delta^{1} F\left(w^{n}\right) \backslash\right.$ 
$\left.\partial \Delta_{F\left(w^{n}\right)}^{1}\right)^{n-1}$. Thus, $\pi_{0}\left(f_{n} E\right)(F)$ is generated by compositions in the diagram

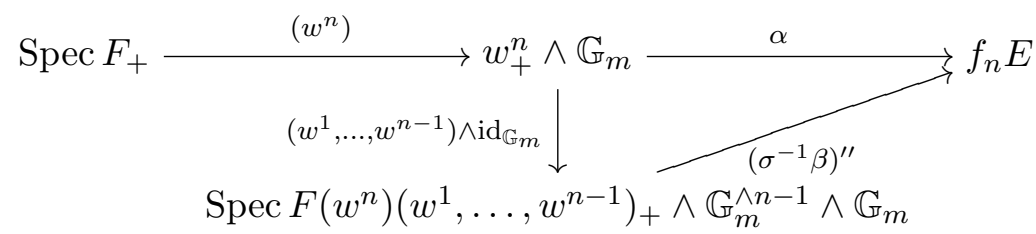

We have the canonical $k$-isomorphism

$$
i:\left(w^{1}, \ldots, w^{n-1}, w^{n}\right) \rightarrow \operatorname{Spec} F\left(w^{n}\right)\left(w^{1}, \ldots, w^{n-1}\right),
$$

so we can fill in this diagram to give the commutative diagram

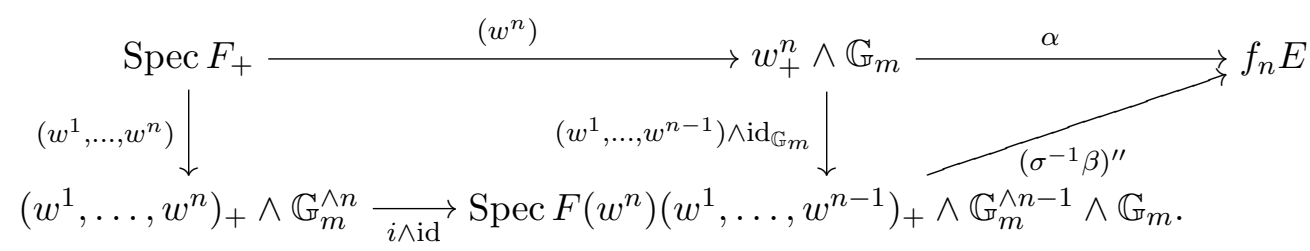

This expresses $\tilde{\epsilon}_{-1}(\alpha)$ as a sum of elements of the form $\gamma^{\prime \prime} \circ\left(w^{1}, \ldots, w^{n}\right)$, with $\gamma \in \pi_{0}\left(\left(\Omega_{\mathbb{G}_{m}}^{n} E\right)\left(w^{1}, \ldots, w^{n}\right)\right)$, and $\left(w^{1}, \ldots, w^{n}\right)$ a closed point of $\left(\Delta_{F}^{1} \backslash \partial \Delta_{F}^{1}\right)^{n}$, as desired.

Composing the map $\tilde{\epsilon}_{-n}$ with the canonical map $\tilde{f}_{n} E \rightarrow E$ yields our description of generators for $F_{\text {Tate }}^{n} \pi_{0} E(F)$.

Proposition 4.3. Let $F$ be a perfect field extension of $k$ and let $E \in \mathbf{S p t}_{S^{1}}(k)$ be quasi-fibrant.

1. Let $w$ be a closed point of $\left(\Delta_{F}^{1} \backslash \partial \Delta_{F}^{1}\right)^{n}$, and take $\alpha_{w} \in \pi_{0}\left(\Omega_{\mathbb{G}_{m}}^{n} E(w)\right)$. Then $\alpha_{w}^{\prime} \circ(w)$ is in $F_{\text {Tate }}^{n} \pi_{0} E(F)$.

2. Suppose that $\Pi_{a, *} E=0$ for all $a<0$. Then $F_{\text {Tate }}^{n} \pi_{0} E(F)$ is generated by elements of the form $\alpha_{w}^{\prime} \circ(w), \alpha_{w} \in \pi_{0}\left(\Omega_{\mathbb{G}_{m}}^{n} E(w)\right)$, as $w$ runs over closed points of $\left(\Delta_{F}^{1} \backslash \partial \Delta_{F}^{1}\right)^{n}$. 


\section{Transfers And $\mathbb{P}^{1}$-SUSPEnsion}

We define a transfer map adapted to our situation. The contractibility of $\mathbb{A}^{1}$ gives the isomorphism in $\mathcal{H}_{\bullet}(k)$

$$
\mathbb{A}^{1} / \mathbb{A}^{1} \backslash\{0\} \cong \Sigma_{s} \mathbb{G}_{m} ;
$$

putting this together with the isomorphisms (4.3) gives us inductively the isomorphism

$$
\tilde{\alpha}_{n}: \mathbb{A}^{n} / \mathbb{A}^{n} \backslash\{0\} \rightarrow\left(\Sigma_{s} \mathbb{G}_{m}\right)^{\wedge n} .
$$

Combining with the "shuffle" isomorphism $\left(\Sigma_{s} \mathbb{G}_{m}\right)^{\wedge n} \rightarrow \Sigma_{s}^{n} \mathbb{G}_{m}^{\wedge n}$ defines the isomorphism

$$
\alpha_{n}: \mathbb{A}^{n} / \mathbb{A}^{n} \backslash\{0\} \rightarrow \Sigma_{s}^{n} \mathbb{G}_{m}^{\wedge n}
$$

in $\mathcal{H}_{\bullet}(k)$; for $U \in \mathbf{S m} / k$, this gives us

$$
\alpha_{n, U}: \mathbb{A}_{U}^{n} / \mathbb{A}_{U}^{n} \backslash\left\{0_{U}\right\} \rightarrow \Sigma_{s}^{n} \mathbb{G}_{m}^{\wedge n} \wedge U_{+}
$$

via the canonical isomorphism $\mathbb{A}_{U}^{n} / \mathbb{A}_{U}^{n} \backslash\left\{0_{U}\right\} \cong \mathbb{A}^{n} / \mathbb{A}^{n} \backslash\{0\} \wedge U_{+}$. For $F$ a finitely generated field extension of $k$, this yields the isomorphism

$$
\alpha_{n, F}: \mathbb{A}_{F}^{n} / \mathbb{A}_{F}^{n} \backslash\left\{0_{F}\right\} \rightarrow \Sigma_{s}^{n} \mathbb{G}_{m}^{\wedge n} \wedge \operatorname{Spec} F_{+}
$$

in $\mathcal{H}_{\bullet}(k)$.

Let $F$ be a finitely generated field extension of $k$, and $w \in \mathbb{A}_{F}^{n}$ a closed point, with $F(w)$ separable over $F$. The maps $\operatorname{id}_{w}, i_{w}: w \rightarrow \mathbb{A}_{F}^{n}$ induce the canonical point $\tilde{i}_{w}: w \rightarrow \mathbb{A}_{F(w)}^{n}$. We let

$$
p: \mathbb{A}_{F(w)}^{n} \rightarrow \mathbb{A}_{F}^{n}
$$

be the projection. We let

$$
T_{w}: \mathbb{A}_{F(w)}^{n} \rightarrow \mathbb{A}_{F(w)}^{n}
$$

denote translation by $w$.

We define an isomorphism

$$
\alpha_{w}: \mathbb{A}_{F}^{n} / \mathbb{A}_{F}^{n} \backslash w \rightarrow \Sigma_{s}^{n} \mathbb{G}_{m}^{\wedge n} \wedge w_{+}
$$

as the composition

$$
\mathbb{A}_{F}^{n} / \mathbb{A}_{F}^{n} \backslash w \stackrel{p}{\sim} \mathbb{A}_{F(w)}^{n} / \mathbb{A}_{F}^{n} \backslash \tilde{i}_{w}(w) \stackrel{T_{-w}}{\longrightarrow} \mathbb{A}_{F(w)}^{n} / \mathbb{A}_{F(w)}^{n} \backslash 0 \stackrel{\alpha_{n, F}}{\longrightarrow} \Sigma_{s}^{n} \mathbb{G}_{m}^{\wedge n} \wedge w_{+} .
$$

Here $p: \mathbb{A}_{F(w)}^{n} / \mathbb{A}_{F}^{n} \backslash \tilde{i}_{w}(w) \rightarrow \mathbb{A}_{F}^{n} / \mathbb{A}_{F}^{n} \backslash w$ is an isomorphism by Nisnevich excision. 
Let $j: \mathbb{A}^{n} \rightarrow \mathbb{P}^{n}$ be the standard open immersion identifying $\mathbb{A}^{n}$ with the open subscheme $X_{0} \neq 0$, and let $H \subset \mathbb{P}^{n}$ be the complement $X_{0}=0$. We note that the quotient map

$$
\pi_{0}^{\prime}: \mathbb{P}^{n} / H \rightarrow \mathbb{P}^{n} / \mathbb{P}^{n} \backslash 0
$$

is an isomorphism in $\mathcal{H}_{\bullet}(k)$, as the linear projection from 0 makes $\mathbb{P}^{n} \backslash 0 \rightarrow H$ an $\mathbb{A}^{1}$-bundle. The transfer map

$$
\operatorname{Tr}_{F}(w): \Sigma_{s}^{n} \mathbb{G}_{m}^{\wedge n} \wedge \operatorname{Spec} F_{+} \rightarrow \Sigma_{s}^{n} \mathbb{G}_{m}^{\wedge n} \wedge w_{+}
$$

is defined as the composition

$$
\begin{aligned}
\Sigma_{s}^{n} \mathbb{G}_{m}^{\wedge n} \wedge \operatorname{Spec} F_{+} & \stackrel{\alpha_{0}}{\sim} \mathbb{A}_{F}^{n} / \mathbb{A}_{F}^{n} \backslash 0 \\
& \stackrel{j}{\sim} \mathbb{P}_{F}^{n} / \mathbb{P}_{F}^{n} \backslash 0 \\
& \stackrel{\pi_{0}^{\prime}}{\sim} \mathbb{P}_{F}^{n} / H_{F} \\
& \stackrel{\pi_{w}^{\prime}}{\longrightarrow} \mathbb{P}_{F}^{n} / \mathbb{P}_{F}^{n} \backslash w \\
& \stackrel{j}{\sim} \mathbb{A}_{F}^{n} / \mathbb{A}_{F}^{n} \backslash w \\
& \stackrel{\alpha_{w}}{\sim} \Sigma_{s}^{n} \mathbb{G}_{m}^{\wedge n} \wedge w_{+},
\end{aligned}
$$

with $\pi_{w}^{\prime}$ the quotient map. We let $\beta_{F}^{n}$ denote the isomorphism

$$
\alpha_{0} \circ j \circ \pi_{0}^{\prime}: \Sigma_{s}^{n} \mathbb{G}_{m}^{\wedge n} \wedge \operatorname{Spec} F_{+} \rightarrow \mathbb{P}_{F}^{n} / H_{F} .
$$

in $\mathcal{H}_{\bullet}(k)$, and let $M V_{w}^{\prime}: \mathbb{P}_{F}^{n} / \mathbb{P}_{F}^{n} \backslash w \rightarrow \Sigma_{s}^{n} \mathbb{G}_{m}^{\wedge n} \wedge w_{+}$denote the isomorphism

$$
\alpha_{w} \circ j: \mathbb{P}_{F}^{n} / \mathbb{P}_{F}^{n} \backslash w \rightarrow \Sigma_{s}^{n} \mathbb{G}_{m}^{\wedge n} \wedge w_{+}
$$

in $\mathcal{H}_{\bullet}(k)$.

For $w$ an $F$-rational point of $\mathbb{A}_{F}^{n}$, the projection to $\operatorname{Spec} F$ defines a canonical isomorphism $q: w \rightarrow \operatorname{Spec} F$.

Lemma 5.1. For $w \in \mathbb{A}^{n}(F)$, the map

$$
(\mathrm{id} \wedge q) \circ \operatorname{Tr}_{F}(w): \Sigma_{s}^{n} \mathbb{G}_{m}^{\wedge n} \wedge \operatorname{Spec} F_{+} \rightarrow \Sigma_{s}^{n} \mathbb{G}_{m}^{\wedge n} \wedge \operatorname{Spec} F_{+}
$$

is the identity. 
Proof. For $w=0$, this follows directly from the definition. In general, the translation map $T_{w}$, extends canonically to the morphism $\bar{T}_{w}:\left(\mathbb{P}^{n}, H\right) \rightarrow\left(\mathbb{P}^{n}, H\right)$, and it suffices to show that $\bar{T}_{w}: \mathbb{P}_{F}^{n} / H_{F} \rightarrow \mathbb{P}_{F}^{n} / H_{F}$ is the identity in $\mathcal{H}_{\bullet}(k)$. This follows from the fact that we may form the $\mathbb{A}^{1}$ family of morphisms $\bar{T}_{w}(t):=\bar{T}_{t \cdot w}$, with $\bar{T}_{w}(0)=$ id, $\bar{T}_{w}(1)=\bar{T}_{w}$.

\section{Computing the collapse map}

In this section we consider the "Pontryagin-Thom collapse map" (4.2)

$$
P T_{F}(w):=M V_{w} \circ \pi_{w} \circ \sigma_{F}^{n}: \Sigma_{s}^{n} \operatorname{Spec} F_{+} \rightarrow w_{+} \wedge \Sigma_{s}^{n} \mathbb{G}_{m}^{\wedge n},
$$

defined in section 4 for $w$ a closed point of $\left(\Delta_{F}^{1} \backslash \partial \Delta_{F}^{1}\right)^{n}$, separable over $F$. We first consider the case of an $F$-rational point, and $n=1$.

For $w \in F^{\times}$, we have the corresponding morphism $w: \operatorname{Spec} F \rightarrow \mathbb{A}^{1} \backslash\{0\}$ and the map (id, $w): \operatorname{Spec} F \rightarrow \operatorname{Spec} F \times{ }_{k} \mathbb{A}^{1} \backslash\{0\}$. This latter map induces the morphism

$$
[w]: \operatorname{Spec} F_{+} \rightarrow \operatorname{Spec} F_{+} \wedge \mathbb{G}_{m}
$$

in $\mathcal{H}_{\bullet}(k)$.

Lemma 6.1. For $w=\left(w_{0}, w_{1}\right)$ an F-point of $\Delta^{1} \backslash \partial \Delta^{1}$, we have

$$
P T_{F}(w)=\Sigma_{s}\left[-w_{1} / w_{0}\right]
$$

Proof. The coboundary maps $\delta_{0}, \delta_{1}: *=\operatorname{Spec} k \rightarrow \Delta^{1}$ have

$$
\delta_{0}(*)=(0,1), \delta_{1}(*)=(1,0)
$$

so the canonical isomorphism $\sigma_{F}^{1}: \Sigma_{s} \operatorname{Spec} F_{+} \rightarrow \operatorname{Spec} F_{+} \wedge \Delta^{1} / \partial \Delta^{1}$ is induced by an isomorphism

$$
([0,1], 0,1) \rightarrow\left(\Delta^{1},(0,1),(1,0)\right)
$$

in $\mathcal{H}_{\bullet}(k)$. Referring to the diagram (4.1), we see that the composition (4.2) is equal to the suspension of the map

$$
\left[-w_{1} / w_{0}\right]: \operatorname{Spec} F_{+} \rightarrow \operatorname{Spec} F_{+} \wedge \mathbb{G}_{m}
$$

as claimed.

This extends easily to the $n$-variable case. 
Proposition 6.2. Let $w=\left(w^{1}, \ldots, w^{n}\right)$ be an F-point of $\left(\Delta^{1} \backslash \partial \Delta^{1}\right)^{n}$, with $w^{i}=\left(w_{0}^{i}, w_{1}^{i}\right)$. Suppose that $F(w)$ is separable over $F$. Then

$$
P T_{F}(w)=\Sigma_{s}^{n}\left(\left[-w_{1}^{1} / w_{0}^{1}\right] \wedge_{F} \ldots \wedge_{F}\left[-w_{1}^{n} / w_{0}^{n}\right]\right) .
$$

Here, the iterated $\wedge_{F}$ means the composition of the smash product of the maps $\left[-w_{1}^{1} / w_{0}^{1}\right] \wedge \ldots \wedge\left[-w_{1}^{n} / w_{0}^{n}\right]$ with the diagonal $\operatorname{Spec} F_{+} \rightarrow \operatorname{Spec} F_{+} \wedge \ldots \wedge \operatorname{Spec} F_{+}$.

Proof. Since $w$ is $F$-rational, $M V_{w}=X_{w}$. By construction of the maps $X_{w}$ and $\sigma_{F}^{n}$, we have

$$
M V_{w} \circ \pi_{w} \circ \sigma_{F}^{n}=\left(M V_{w^{1}} \circ \pi_{w^{1}} \circ \sigma_{F}^{1}\right) \wedge_{F} \ldots \wedge_{F}\left(M V_{w^{n}} \circ \pi_{w^{n}} \circ \sigma_{F}^{1}\right) .
$$

Thus, the result follows directly from lemma 6.1.

For $w \in\left(\Delta_{F}^{1} \backslash \partial \Delta_{F}^{1}\right)^{n}$, separable over $F$, there is in general no nice formula for the composition $M V_{w} \circ \pi_{w} \circ \sigma_{F}^{n}$, however, using the construction of transfers in section 5 , we have a suitable extension of our computation.

To simplify the notation, we write $S^{a+b, b}$ for $\Sigma_{s}^{a} \mathbb{G}_{m}^{\wedge b}$, so for instance $S^{2 n, n}=$ $\Sigma_{s}^{n} \mathbb{G}_{m}^{\wedge n}, S^{n, 0}=\Sigma_{s}^{n} \operatorname{Spec} k_{+}=S^{n}$.

Let $\rho_{1}: \Delta^{1} \backslash \partial \Delta^{1} \rightarrow \mathbb{A}^{1}$ be the open immersion

$$
\rho_{1}\left(t_{0}, t_{1}\right)=-\frac{t_{1}}{t_{0}}
$$

identifying $\Delta^{1} \backslash \partial \Delta^{1}$ with $\mathbb{A}^{1} \backslash\{0,1\}$. Taking the $n$-fold product of the $\rho_{1}$ gives us the open immersion

$$
\rho_{n}:\left(\Delta^{1} \backslash \partial \Delta^{1}\right)^{n} \rightarrow \mathbb{A}^{n}
$$

Proposition 6.3. Let $w=\left(w^{1}, \ldots, w^{n}\right)$ be a closed point of $\left(\Delta_{F}^{1} \backslash \partial \Delta_{F}^{1}\right)^{n}$, separable over $F$. Write $w^{i}=\left(w_{0}^{i}, w_{1}^{i}\right)$. Then the $S^{2 n, n}$-suspension of $\operatorname{PT}_{F}(w)$ :

$$
\operatorname{id}_{S^{2 n, n}} \wedge P T_{F}(w): S^{2 n, n} \wedge \operatorname{Spec} F_{+} \wedge S^{n, 0} \rightarrow S^{2 n, n} \wedge w_{+} \wedge S^{2 n, n}
$$

is equal to the map

$$
\Sigma_{s}^{n}\left(\left(\operatorname{id}_{S^{2 n, n}} \wedge\left[-w_{1}^{1} / w_{0}^{1}\right] \wedge_{F(w)} \cdots \wedge_{F(w)}\left[-w_{1}^{n} / w_{0}^{n}\right]\right) \circ \operatorname{Tr}_{F}\left(\rho_{n}(w)\right)\right) .
$$

Proof. Write $*_{F}$ for $\operatorname{Spec} F$ and $w^{\prime}$ for $\rho_{n}(w)$. We identify the points $w$ and $w^{\prime}$ by the isomorphism $\rho$, but avoid writing in the $\rho$ to simplify the notation. We 
have the commutative diagram

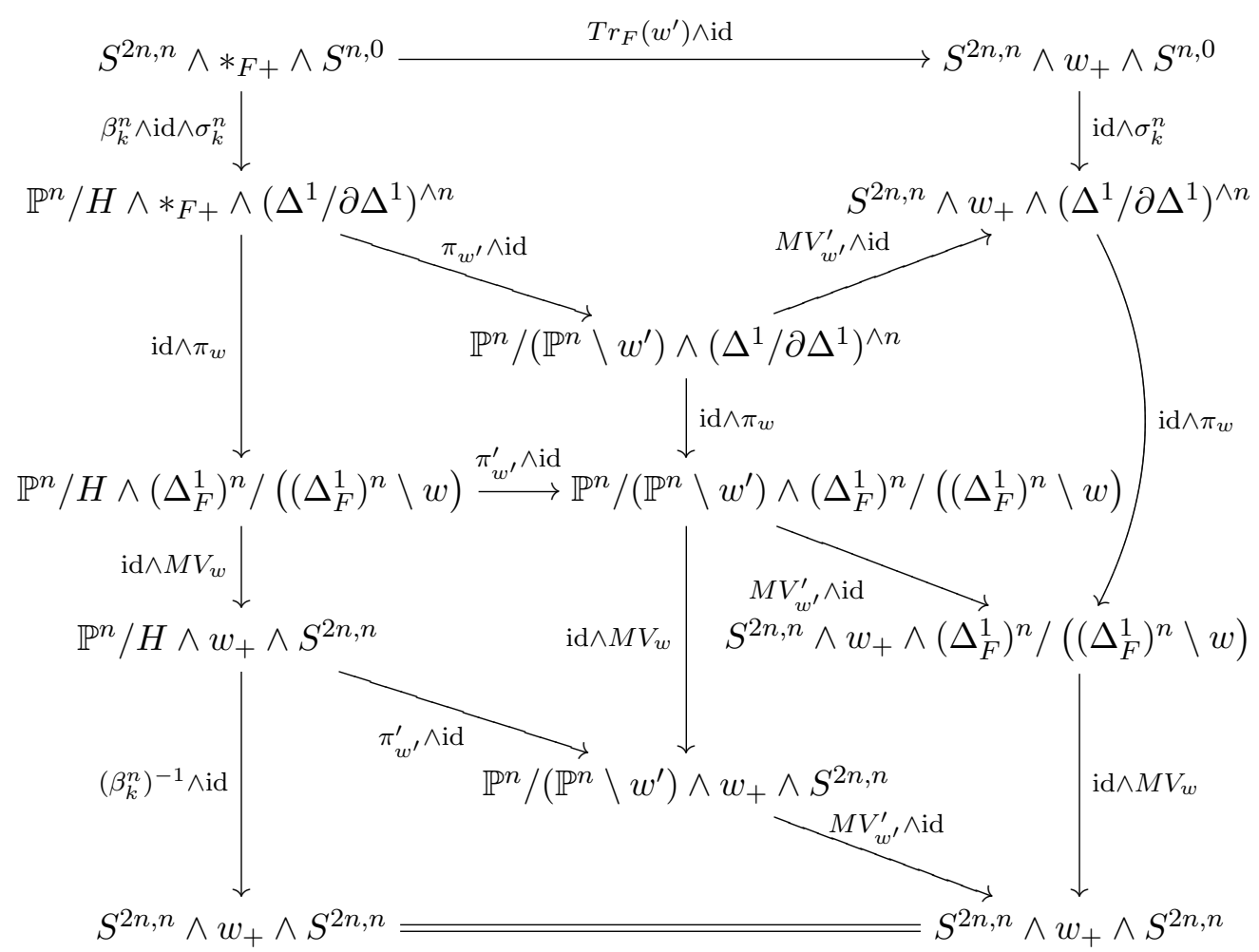

the commutativity follows either by definition of $\operatorname{Tr}_{F}(w)$, or by identities of the form $(a \wedge 1) \circ(1 \wedge b)=(1 \wedge b) \circ(a \wedge 1)$, or (in the bottom square) lemma 5.1. The composition along the left-hand side is $\operatorname{id}_{S^{2 n, n}} \wedge P T_{F}(w)$; along the righthand side we have $\left.\operatorname{id}_{S^{2 n, n}} \wedge P T_{F(w)}(w)\right]$. Since $w$ is $F(w)$-rational, we may apply proposition 6.2 to complete the proof.

\section{Conclusion}

We can now put all the pieces together. For $E \in \mathbf{S p t}_{S^{1}}(k)$ fibrant, we have the associated fibrant object $\Omega_{T}^{n} E:=\mathcal{H o m}_{\mathbf{S p t}(k)}\left(S^{2 n, n}, E\right)$, that is, $\Omega_{T}^{n} E$ is the presheaf $\left(\Omega_{T}^{n} E\right)(X):=E\left(X_{+} \wedge S^{2 n, n}\right)$. For each $n \geq 1$, we have the canonical map

$$
\iota_{n}: E \rightarrow \Omega_{T}^{n} \Sigma_{T}^{n} E .
$$

Replacing $S^{2 n, n}$ with $S^{n, n}=\mathbb{G}_{m}^{\wedge n}$, we have the fibrant object

$$
\Omega_{\mathbb{G}_{m}}^{n} E:=\mathcal{H}_{\mathrm{Som}} \operatorname{Spt(k)}\left(S^{n, n}, E\right),
$$


defined as the presheaf $\left(\Omega_{\mathbb{G}_{m}}^{n} E\right)(X):=E\left(X_{+} \wedge \mathbb{G}_{m}^{\wedge n}\right)$.

Let $V_{n}$ denote the product $\left(\Delta^{1} \backslash \partial \Delta^{1}\right)^{n}$. Recall the open immersion

$$
\rho_{n}: V_{n} \rightarrow \mathbb{A}^{n}
$$

identifying $V_{n}$ with $\left(\mathbb{A}^{1} \backslash\{0,1\}\right)^{n} \subset \mathbb{A}^{n}$. As before, we denote $\rho_{n}(w)$ by $w^{\prime}$ and identify $w$ and $w^{\prime}$ via $\rho_{n}$.

Given a closed point $w \in V_{n F}$, we define the map

$$
\operatorname{Tr}_{F}(w)^{*}: \pi_{m}\left(\Omega_{T}^{n} E(w)\right) \rightarrow \pi_{m}\left(\Omega_{T}^{n} E(F)\right)
$$

as the composition

$$
\begin{aligned}
\pi_{m}\left(\Omega_{T}^{n} E(w)\right) & =\operatorname{Hom}_{\mathcal{S H}_{S^{1}}(k)}\left(\Sigma_{s}^{\infty}\left(S^{2 n, n} \wedge w_{+}\right), \Sigma_{s}^{-m} E\right) \\
& \stackrel{\left.\Sigma_{s}^{\infty}\left(\operatorname{Tr}_{F}\left(w^{\prime}\right)\right)\right)^{*}}{\longrightarrow} \operatorname{Hom}_{\mathcal{S H}_{S^{1}}(k)}\left(\Sigma_{s}^{\infty}\left(S^{2 n, n} \wedge \operatorname{Spec} F_{+}\right), \Sigma_{s}^{-m} E\right) \\
& =\pi_{m}\left(\Omega_{T}^{n} E(F)\right) .
\end{aligned}
$$

Definition 7.1. Take $E \in \mathcal{S H}_{S^{1}}(k)$ and let $n \geq 1$ be an integer. An $n$-fold $T$-delooping of $E$ is an an object $\omega_{T}^{-n} E$ of $\mathcal{S H}_{S^{1}}(k)$ and an isomorphism $\iota_{n}: E \rightarrow$ $\Omega_{T}^{n} \omega_{T}^{-n} E$ in $\mathcal{S H}_{S^{1}}(k)$.

Given an $n$-fold $T$-delooping of $E, \iota_{n}: E \rightarrow \Omega_{T}^{n} \omega_{T}^{-n} E$, the map $\operatorname{Tr}_{F}(w)^{*}$ for $\Omega_{T}^{n} \omega_{T}^{-n} E$ induces the "transfer map"

$$
\iota_{n}^{-1} \circ \operatorname{Tr}_{F}(w)^{*} \circ \iota_{n}: \pi_{m}(E(w)) \rightarrow \pi_{m}(E(F)),
$$

which we write simply as $\operatorname{Tr}_{F}(w)^{*}$.

Remarks 7.2. 1. The transfer map $\operatorname{Tr}_{F}(w)^{*}: \pi_{m}(E(w)) \rightarrow \pi_{m}(E(F))$ may possibly depend on the choice of $n$-fold $T$-delooping, we do not have an example, however.

2. An $n$ - $b$-fold $T$-delooping of $E$ gives rise to an $n$-fold $T$-delooping of $\Omega_{\mathbb{G}_{m}}^{b} E$. Thus, via the adjunction isomorphism

$$
\Pi_{a, b} E \cong \pi_{a} \Omega_{\mathbb{G}_{m}}^{b} E
$$

we have a transfer map

$$
\operatorname{Tr}_{F}(w)^{*}: \Pi_{a, b} E(w) \rightarrow \Pi_{a, b} E(F)
$$


for $w$ a closed point of $V_{n F}$, separable over $F$.

3. If $E=\Omega_{T}^{\infty} \mathcal{E}$ for some $\mathcal{E} \in \mathcal{S H}(k)$, then $E$ admits canonical $n$-fold $T$-deloopings, namely

$$
\omega_{T}^{-n} E:=\Omega_{T}^{\infty} \Sigma_{T}^{n} \mathcal{E} .
$$

Indeed, in $\mathcal{S H}(k), \Sigma_{T}$ is the inverse to $\Omega_{T}$ and $\Omega_{T}^{\infty}$ commutes with $\Omega_{T}$.

For a morphism $\varphi: \Sigma_{s}^{\infty} w_{+} \rightarrow E$, we have the suspension $\Sigma_{T}^{n} \varphi: \Sigma_{T}^{n} \Sigma_{s}^{\infty} w_{+} \rightarrow$ $\Sigma_{T}^{n} E$, the composition

$$
\Sigma_{T}^{n} \varphi \circ \Sigma_{s}^{\infty} \operatorname{Tr}_{F}(w)^{*}: \Sigma_{T}^{n} \Sigma_{s}^{\infty} \operatorname{Spec} F_{+} \rightarrow \Sigma_{T}^{n} E
$$

and the adjoint morphism

$$
\left(\Sigma_{T}^{n} \varphi \circ \Sigma_{s}^{\infty} \operatorname{Tr}_{F}(w)^{*}\right)^{\prime}: \Sigma_{s}^{\infty} \operatorname{Spec} F_{+} \rightarrow \Omega_{T}^{n} \Sigma_{T}^{n} E .
$$

Suppose we have an $n$-fold de-looping of $E, \iota_{n}: E \rightarrow \Omega_{T}^{n} \omega_{T}^{-n} E$. This gives us the adjoint

$$
\iota_{n}^{\prime}: \Sigma_{T}^{n} E \rightarrow \omega_{T}^{-n} E
$$

and

$$
\Omega_{T}^{n} \iota_{n}^{\prime}: \Omega_{T}^{n} \Sigma_{T}^{n} E \rightarrow \Omega_{T}^{n} \omega_{T}^{-n} E .
$$

Let $\delta_{n}: E \rightarrow \Omega_{T}^{n} \sum_{T}^{n} E$ be the unit for the adjunction.

Lemma 7.3. 1. $\iota_{n}=\Omega_{T}^{n} \iota_{n}^{\prime} \circ \delta_{n}$

2. $\iota_{n}^{-1} \circ \Omega_{T}^{n} \iota_{n}^{\prime} \circ\left(\Sigma_{T}^{n} \varphi \circ \Sigma_{s}^{\infty} \operatorname{Tr}_{F}(w)\right)^{\prime}=\operatorname{Tr}_{F}(w)^{*}(\varphi)$.

Proof. The two assertions follow from the universal property of adjunction.

Before proving our main results, we show that the transfer maps respect the Postnikov filtration $F_{\text {Tate }}^{*} \pi_{m} E$.

Lemma 7.4. Suppose $E$ admits an $n$-fold T-delooping $\iota_{n}: E \rightarrow \Omega_{T}^{n} \omega_{T}^{-n} E$. Then for each finitely generated field $F$ over $k$ and each closed point $w \in \mathbb{A}_{F}^{n}$ separable over $F$, we have

$$
\operatorname{Tr}_{F}(w)^{*}\left(F_{\text {Tate }}^{q} \pi_{m} E(w)\right) \subset F_{\text {Tate }}^{q} \pi_{m} E(F) .
$$


Proof. Take $q \geq 0$, and let $\tau_{q}: f_{q} E \rightarrow E$ be the canonical morphism. As above, let $\iota_{n}^{\prime}: \Sigma_{T}^{n} E \rightarrow \omega_{T}^{-n} E$ be the adjoint of $\iota_{n}$ and let $\delta_{n}: E \rightarrow \Omega_{T}^{n} \Sigma_{T}^{n} E$ be the unit of the adjunction. By lemma 7.3, we have the factorization of $\iota_{n}$ as

$$
E \stackrel{\delta_{n}}{\longrightarrow} \Omega_{T}^{n} \Sigma_{T}^{n} E \stackrel{\Omega_{T}^{n} \iota_{n}^{\prime}}{\longrightarrow} \Omega_{T}^{n} \omega_{T}^{-n} E
$$

This gives us the commutative diagram

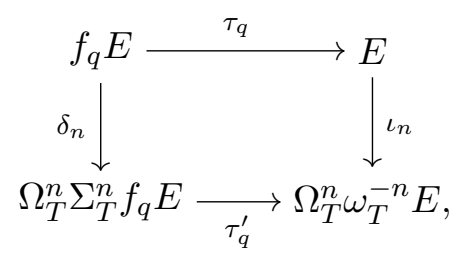

where $\tau_{q}^{\prime}:=\Omega_{T}^{n} \iota_{n}^{\prime} \circ \Omega_{T}^{n} \Sigma_{T}^{n} \tau_{q}$. Since $\iota_{n}: E \rightarrow \Omega_{T}^{n} \omega_{T}^{-n} E$ is an isomorphism, the composition

$$
\iota_{n} \circ \tau_{q}: f_{q} E \rightarrow \Omega_{T}^{n} \omega_{T}^{-n} E
$$

satisfies the universal property of $f_{q} \Omega_{T}^{n} \omega_{T}^{-n} E \rightarrow \Omega_{T}^{n} \omega_{T}^{-n} E$. By [6, theorem 7.4.1], $\Omega_{T}^{n} \Sigma_{T}^{n} f_{q} E$ is in $\Sigma_{T}^{q} \mathcal{S} \mathcal{H}_{S^{1}}(k)$, hence there is a canonical morphism

$$
\theta: \Omega_{T}^{n} \Sigma_{T}^{n} f_{q} E \rightarrow f_{q} E
$$

extending our first diagram to the commutative diagram

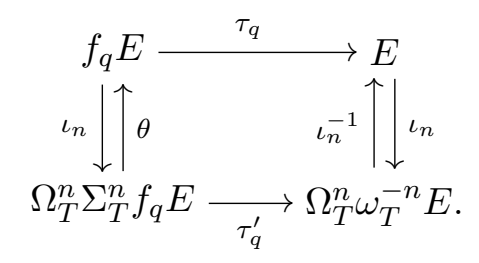

Using the universal property of $\tau_{q}$, we see that $\theta \circ \iota_{n}=\operatorname{id}_{f_{q} E}$, i.e.,

$$
\Omega_{T}^{n} \Sigma_{T}^{n} f_{q} E=f_{q} E \oplus R
$$

and the restriction of $\tau_{q}^{\prime}$ to $R$ is the zero map. We define the transfer map

$$
\operatorname{Tr}_{F}(w)^{*}: \pi_{m} f_{q} E(w) \rightarrow \pi_{m} f_{q} E(F)
$$

by using the transfer map for $\Omega_{T}^{n} \Sigma_{T}^{n} f_{q} E$ and this splitting. 
The second diagram thus gives rise to the commutative diagram

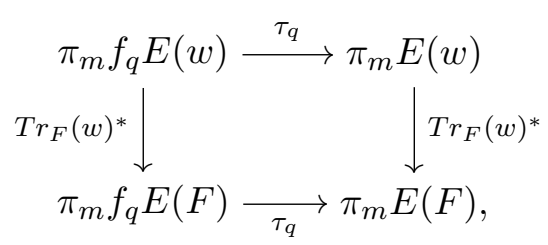

which yields the result.

Remark 7.5. One can define transfer maps in a more general setting, that is, for a closed point $w \in \mathbb{A}_{F}^{n}$ and any choice of parameters for $m_{w} \subset \mathcal{O}_{\mathbb{A}^{n}, w}$. The same proof as used for lemma 7.4 shows that these more general transfer maps respect the filtration $F_{\text {Tate }}^{*} \pi_{m} E$.

Theorem 7.6. Let $E \in \mathbf{S p t}(k)$ be fibrant, and let $F$ be a field extensions of $k$.

1. For each $w=\left(w^{1}, \ldots, w^{n}\right) \in V_{n}(F), w^{i}=\left(w_{0}^{i}, w_{1}^{i}\right)$, and each $\rho \in \pi_{0} \Omega_{\mathbb{G}_{m}}^{n} E(F)$, the element

$$
\rho \circ \Sigma_{s}^{\infty}\left(\left[-w_{1}^{1} / w_{0}^{1}\right] \wedge_{F} \ldots \wedge_{F}\left[-w_{1}^{n} / w_{0}^{n}\right]\right): \Sigma_{s}^{\infty} \operatorname{Spec} F_{+} \rightarrow E
$$

is in $F_{\text {Tate }}^{n} \pi_{0} E(F)$.

2. Suppose that $E$ admits an $n$-fold T-delooping $\iota_{n}: E \rightarrow \Omega_{T}^{n} \omega_{T}^{-n} E$. Then forw $=\left(w^{1}, \ldots, w^{n}\right), w^{i}=\left(w_{0}^{i}, w_{1}^{i}\right)$, a closed point of $V_{n F}$, separable over $F$, and $\rho_{w} \in \pi_{0} \Omega_{\mathbb{G}_{m}}^{n} E(w)$

$$
\operatorname{Tr}_{F}(w)^{*}\left[\rho_{w} \circ \Sigma_{s}^{\infty}\left(\left[-w_{1}^{1} / w_{0}^{1}\right] \wedge_{F} \ldots \wedge_{F}\left[-w_{1}^{n} / w_{0}^{n}\right]\right)\right]
$$

is in $F_{\text {Tate }}^{n} \pi_{0} E(F)$.

3. Suppose that $E$ admits an $n$-fold T-delooping $\iota_{n}: E \rightarrow \Omega_{T}^{n} \omega_{T}^{-n} E$. and that $\Pi_{a, *} E=0$ for all $a<0$. Suppose further that $F$ is perfect. Then $F_{\text {Tate }}^{n} \pi_{0} E(F)$ is generated by elements of the form (7.1), as $w$ runs over closed point of $V_{n F}$ and $\rho_{w}$ over elements of $\pi_{0} \Omega_{\mathbb{G}_{m}}^{n} E(w)$.

Proof. (1) follows directly from proposition 4.3 and proposition 6.2.

For (2), the fact that this element is in $F_{\text {Tate }}^{n} \pi_{0}(E(F))$ follows from (1) and lemma 7.4. 
For (3), that is, to see that these elements generate, take one of the generators $\gamma:=\alpha_{w}^{\prime} \circ(w)$ of $F_{\text {Tate }}^{n} \pi_{0} E(F)$, as given by proposition 4.3. Here $w$ is a closed point of $V_{n F}, \alpha_{w}$ is in $\pi_{0}\left(\Omega_{T}^{n} E(w)\right)$ and $\alpha^{\prime}: w_{+} \wedge \mathbb{G}_{m}^{\wedge n} \rightarrow E$ is the morphism in $\mathcal{S H}_{S^{1}}(k)$ adjoint to $\alpha$. Since $F$ is perfect, $w$ is separable over $F$. Take the $n$-fold $T$-suspension of $\gamma$

$$
\Sigma_{T}^{n} \gamma: \Sigma_{s}^{\infty}\left(\Sigma_{T}^{n} \operatorname{Spec} F_{+}\right) \rightarrow \Sigma_{T}^{n} E
$$

giving by adjunction and composition with $\Omega_{T}^{n}\left(\iota_{n}^{\prime}\right)$ the morphism

$$
\Omega_{T}^{n}\left(\iota_{n}^{\prime}\right) \circ\left(\Sigma_{T}^{n} \gamma\right)^{\prime}: \Sigma_{s}^{\infty} \operatorname{Spec} F_{+} \rightarrow \Omega_{T}^{n} \omega^{-n} E .
$$

It follows from the universal properties of adjunction that

$$
\left(\Sigma_{T}^{n} \gamma\right)^{\prime}=\delta_{n} \circ \gamma
$$

hence by lemma 7.3 we have

$$
\Omega_{T}^{n}\left(\iota_{n}^{\prime}\right) \circ\left(\Sigma_{T}^{n} \gamma\right)^{\prime}=\Omega_{T}^{n}\left(\iota_{n}^{\prime}\right) \circ \delta_{n} \circ \gamma=\iota_{n} \circ \gamma .
$$

Write

$$
\Sigma_{T}^{n} \gamma=\left(\Sigma_{T}^{n} \alpha_{w}^{\prime}\right) \circ\left(\Sigma_{s}^{\infty} \Sigma_{T}^{n}(w)\right) .
$$

By proposition 6.3 we have

$$
\Sigma_{T}^{n}(w)=\left(\Sigma_{T}^{n}\left[-w_{1}^{1} / w_{0}^{1}\right] \wedge_{F} \ldots \wedge_{F}\left[-w_{1}^{n} / w_{0}^{n}\right] \circ \operatorname{Tr}_{F}(w)\right),
$$

and thus

$$
\Sigma_{T}^{n} \gamma=\Sigma_{T}^{n}\left(\alpha_{w}^{\prime} \circ\left[-w_{1}^{1} / w_{0}^{1}\right] \wedge_{F} \ldots \wedge_{F}\left[-w_{1}^{n} / w_{0}^{n}\right]\right) \circ \operatorname{Tr}_{F}(w) .
$$

Using (7.2) and lemma 7.3, we have

$$
\begin{aligned}
\iota_{n} \circ \gamma & =\Omega_{T}^{n}\left(\iota_{n}^{\prime}\right) \circ\left(\Sigma_{T}^{n} \gamma\right)^{\prime} \\
& =\Omega_{T}^{n}\left(\iota_{n}^{\prime}\right) \circ\left[\Sigma_{T}^{n}\left(\xi_{w} \circ \Sigma_{s}^{n}\left[-w_{1} / w_{0}\right] \wedge_{F} \ldots \wedge_{F}\left[-w_{n} / w_{0}\right]\right) \circ \Sigma_{s}^{n} \operatorname{Tr}_{F}(w)\right]^{\prime} \\
& =\iota_{n} \circ \operatorname{Tr}_{F}(w)^{*}\left(\alpha_{w}^{\prime} \circ\left[-w_{1}^{1} / w_{0}^{1}\right] \wedge_{F} \ldots \wedge_{F}\left[-w_{1}^{n} / w_{0}^{n}\right]\right),
\end{aligned}
$$

or

$$
\gamma=\operatorname{Tr}_{F}(w)^{*}\left[\alpha_{w}^{\prime} \circ \Sigma_{s}^{\infty}\left(\left[-w_{1}^{1} / w_{0}^{1}\right] \wedge_{F} \ldots \wedge_{F}\left[-w_{1}^{n} / w_{0}^{n}\right]\right)\right]
$$


We now assume that $E=\Omega_{T}^{\infty} \mathcal{E}$ for some fibrant $T$-spectrum $\mathcal{E} \in \mathbf{S p t}_{T}(k)$. Let $\mathbb{S}_{k}$ denote the motivic sphere spectrum in $\mathbf{S p t}_{T}(k)$, that is, $\mathbb{S}_{k}$ is a fibrant model of the suspension spectrum $\Sigma_{T}^{\infty} S_{k}^{0}$. We proceed to re-interpret theorem 7.6 in terms of the canonical action of $\pi_{0} \Omega_{T}^{\infty} \mathbb{S}_{k}(F)$ on $\pi_{0} E(F)$, which we now recall, along with some of the fundamental computations of Morel relating the Grothendieck-Witt group with endomorphisms of the motivic sphere spectrum.

We recall the Milnor-Witt sheaves of Morel, $\underline{K}_{n}^{M W}$ (see [8, section 2] for details). The graded sheaf $\underline{K}_{*}^{M W}:=\oplus_{n \in \mathbb{Z}} \underline{K}_{n}^{M W}$ has structure of a Nisnevich sheaf of associative graded rings. For a field $F$, the graded $\operatorname{ring} K_{*}^{M W}(F):=\underline{K}_{*}^{M W}(F)$ has generators $[u]$ in degree 1 , for $u \in F^{\times}$, and an additional generator $\eta$ in degree -1 , with relations

- $\eta[u]=[u] \eta$

- $[u][1-u]=0$ (Steinberg relation)

- $[u v]=[u]+[v]+\eta[u][v]$

- $\eta(2+\eta[-1])=0$.

For $u \in F^{\times}$, let $\left\langle u>\right.$ denote the quadratic form $u y^{2}$ in the Grothendieck-Witt group $\mathrm{GW}(F)$. Sending $[u] \eta$ to $\langle u>-1$ extends to an isomorphism $[8$, lemma $2.10]$

$$
\vartheta_{0}: K_{0}^{M W}(F) \rightarrow \mathrm{GW}(F) .
$$

In addition, for $n \geq 1$, the image of $\times \eta^{n}: K_{n}^{M W}(F) \rightarrow K_{0}^{M W}(F)$ is an ideal $\eta^{n} K_{n}^{M W}(F)$ in $K_{0}^{M W}(F)$ and $\vartheta_{0}$ maps $\eta^{n} K_{n}^{M W}(F)$ isomorphically onto the ideal $I(F)^{n}$, where $I(F) \subset \mathrm{GW}(F)$ is the augmentation ideal of quadratic forms of virtual rank zero.

For each $u \in F^{\times}$, we have the corresponding morphism

$$
[u]: \operatorname{Spec} F_{+} \rightarrow \mathbb{G}_{m}
$$

We have as well the canonical projection $\eta^{\prime}: \mathbb{A}^{2} \backslash\{0\} \rightarrow \mathbb{P}^{1}$. Using a construction similar to the one we used to show that $\mathbb{P}^{2} / H \cong \Sigma_{s}^{2} \mathbb{G}_{m}^{\wedge 2}$, one constructs a canonical isomorphism in $\mathcal{H}_{\bullet}(k),\left(\mathbb{A}^{2} \backslash\{0\}, 1\right) \cong \Sigma_{s}^{1} \mathbb{G}_{m}^{\wedge 2}$, and thus $\eta^{\prime}$ yields the morphism

$$
\eta: \Sigma_{s}^{1} \mathbb{G}_{m}^{\wedge 2} \rightarrow \Sigma_{s}^{1} \mathbb{G}_{m}
$$

in $\mathcal{H}_{\bullet}(k)$. 
For $E, F \in \mathbf{S p t}_{S^{1}}(k)$, let $\underline{\operatorname{Hom}}(E, F)$ denote the Nisnevich sheaf associated to the presheaf

$$
U \mapsto \operatorname{Hom}_{\mathcal{S H}_{S^{1}}(k)}\left(U_{+} \wedge E, F\right)
$$

We have the fundamental theorem of Morel:

Theorem 7.7 ([8, corollary 3.43]). Suppose char $k \neq 2$. Let $m, p, q \geq 0, n \geq 2$ be integers. Then sending $[u] \in K_{1}^{M W}(F)$ to the morphism $[u]$ and sending $\eta \in K_{-1}^{M W}(F)$ to the morphism $\eta$ yields isomorphisms

$\operatorname{Hom}_{\mathcal{H} \bullet(k)}\left(\operatorname{Spec} F_{+} \wedge S^{m} \wedge \mathbb{G}_{m}^{\wedge p}, S^{n} \wedge \mathbb{G}_{m}^{\wedge q}\right) \cong \begin{cases}0 & \text { if } m<n \\ K_{q-p}^{M W}(F) & \text { if } m=n \text { and } q>0\end{cases}$

As we will be relying on Morel's theorem, we assume for the rest of the paper that the characteristic of $k$ is different from two.

Passing to the $S^{1}$-stabilization, theorem 7.7 gives

$$
\begin{array}{lr}
\Pi_{0, p} \Sigma_{s}^{\infty} \mathbb{G}_{m}^{\wedge q}=\underline{K}_{q-p}^{M W} & \text { for } p \geq 0, q \geq 1, \\
\Pi_{a, p} \Sigma_{s}^{\infty} \mathbb{G}_{m}^{\wedge q}=0 & \text { for } p \geq 0, q \geq 1, a<0 .
\end{array}
$$

Passing to the $T$-stable setting, Morel's theorem gives

$$
\begin{array}{lr}
\pi_{p, p} \Sigma_{\mathbb{G}_{m}}^{q} \mathbb{S}_{k} \cong \underline{K}_{q-p}^{M W} & \text { for } p, q \in \mathbb{Z} \\
\pi_{a+p, p} \Sigma_{\mathbb{G}_{m}}^{q} \mathbb{S}_{k}=0 & \text { for } p, q \in \mathbb{Z}, a<0 .
\end{array}
$$

Composition of morphisms gives us the (right) action of the bi-graded sheaf of rings $\pi_{*, *} \mathbb{S}_{k}$ on $\pi_{*, *} \mathcal{E}$ for each $T$-spectrum $\mathcal{E}$, and thus, the action of $\underline{K}_{-*}^{M W}$ on $\pi_{*, *} \mathcal{E}$. If we let $E$ be the $S^{1}$-spectrum $\Omega_{T}^{\infty} \mathcal{E}$, then $\Pi_{a, b} E=\pi_{a+b, b} \mathcal{E}$ for all $b \geq 0$. Thus, via lemma 2.2(2) we thus have the right multiplication

$$
\Pi_{a, b-m} E \otimes \underline{K}_{-m}^{M W} \rightarrow \Pi_{a, b} E .
$$

Let $\mathcal{I} \subset \underline{K}_{0}^{M W}$ be the sheaf of augmentation ideals. The $\underline{K}_{-*}^{M W}$-module structure on $\Pi_{a, *} E$ gives us the filtration $F_{M W}^{n} \Pi_{a, b} E$ of $\Pi_{a, b} E$, defined by

$$
F_{M W}^{n} \Pi_{a, b} E:=\operatorname{im}\left[\Pi_{a, n} E \otimes \underline{K}_{n-b}^{M W} \rightarrow \Pi_{a, b} E\right] ; \quad n \geq 0 .
$$


Lemma 7.8. Suppose $E=\Omega_{T}^{\infty} \mathcal{E}$ for some $\mathcal{E} \in \mathcal{S H}(k)$. For integers $n, b, p \geq 0$, with $n-p, b-p \geq 0$, the adjunction isomorphism $\Pi_{a, b} E \cong \Pi_{a, b-p} \Omega_{\mathbb{G}_{m}}^{p} E$ induces an isomorphism

$$
F_{M W}^{n} \Pi_{a, b} E \cong F_{M W}^{n-p} \Pi_{a, b-p} \Omega_{\mathbb{G}_{m}}^{p} E
$$

Proof. This follows easily from the fact that the adjunction isomorphism

$$
\Pi_{a, *} E \cong \Pi_{a, *-p} \Omega_{\mathbb{G}_{m}}^{p} E
$$

is a $\underline{K}_{*}^{M W}$-module isomorphism.

Definition 7.9. Let $E=\Omega_{T}^{\infty} \mathcal{E}$ for some $\mathcal{E} \in \mathcal{S H}(k), F$ a field extension of $k$. Take integers $a, b, n$ with $n, b \geq 0$. Following remark 7.2(2), we have the transfer maps

$$
\operatorname{Tr}_{F}(w): \Pi_{a, b} E(F(w)) \rightarrow \Pi_{a, b} E(F)
$$

for each closed point $w \in V_{n F}$, separable over $F$.

1. Let $F_{M W^{T r}}^{n} \Pi_{a, b} E(F)$ denote the subgroup of $\Pi_{a, b} E(F)$ generated by elements of the form

$$
\operatorname{Tr}_{F}(w)^{*}(x) ; \quad x \in F_{M W}^{n} \Pi_{a, b} E(F(w))
$$

as $w$ runs over closed points of $\mathbb{A}_{F}^{n}$, separable over $F$.

2. Let $\left[\Pi_{a, b} E \cdot \mathcal{I}^{n}\right]^{\operatorname{Tr}}(F)$ denote the subgroup of $\Pi_{a, b} E(F)$ generated by elements of the form

$$
\operatorname{Tr}_{F}(w)^{*}(x \cdot y) ; \quad x \in \Pi_{a, b} E(F(w)), y \in I(F(w))^{n},
$$

as $w$ runs over closed points of $\mathbb{A}_{F}^{n}$, separable over $F$.

Remark 7.10. It follows directly from the definitions that, for $w$ a closed point of $V_{n F}, x \in K_{n-b}^{M W}(F), y \in \Pi_{a, n} E(F(w))$, we have

$$
\operatorname{Tr}_{F}(w)^{*}\left(y \cdot p^{*} x\right)=\operatorname{Tr}_{F}(w)^{*}(y) \cdot x,
$$

where $p^{*} x \in K_{n-b}^{M W}(F(w))$ is the extension of scalars of of $x$. In particular, $\left[\Pi_{a, b} E\right.$. $\left.\mathcal{I}^{n}\right]^{T r}(F)$ is a $K_{0}^{M W}(F)$-submodule of $\Pi_{a, b} E(F)$ containing $\Pi_{a, b} E(F) I(F)^{n}$.

Theorem 7.11. Let $k$ be a perfect field of characteristic $\neq 2$. Let $E=\Omega_{T}^{\infty} \mathcal{E}$ for some $\mathcal{E} \in \mathcal{S H}(k)$ with $\Pi_{a, b} \mathcal{E}=0$ for all $a<0, b \geq 0$. Let $n>p \geq 0$ be integers and let $F$ be a perfect field extension of $k$. Then

$$
F_{\text {Tate }}^{n} \Pi_{0, p} E(F)=F_{M W^{T r}}^{n} \Pi_{0, p} E(F) .
$$


For $p \geq n \geq 0$, we have the identity of sheaves $F_{\text {Tate }}^{n} \Pi_{0, p} E=\Pi_{0, p} E$.

Proof. First suppose $n>p$. By lemma 2.2 and lemma 7.8, we reduce to the case $p=0$.

The fact that we have an inclusion of $K_{0}^{M W}(F)$-submodules of $\Pi_{0,0} E(F)$,

$$
F_{\text {Tate }}^{n} \Pi_{0,0} E(F) \subset F_{M W^{T r}}^{n} \Pi_{0,0} E(F),
$$

follows from theorem 7.6. Indeed, as $F$ is perfect, each element of the form (7.1) is of the form $\operatorname{Tr}_{F}(w)\left(\rho_{w} \cdot z\right)$, with $\rho_{w} \in \Pi_{0, n} E(w), z \in K_{n}^{M W}(F(w))$, hence in $F_{M W^{T r}}^{n} \Pi_{0,0} E(F)$.

To show the other inclusion, it suffices by lemma 7.4 and theorem 7.6 to show that, for each field $K$ finitely generated over $k$, the elements $\left[-u_{1}^{1} / u_{0}^{1}\right] \cdot \ldots$. $\left[-u_{1}^{n} / u_{0}^{n}\right]$, with $\left(u^{1}, \ldots, u^{n}\right) \in V_{n}(K), u^{i}=\left(u_{0}^{i}, u_{1}^{i}\right)$, generate $K_{n}^{M W}(K)$ as a module over $K_{0}^{M W}(K)$. But this is clear, as $K_{n}^{M W}(K)$ is generated as a $K_{0}^{M W}(K)$ module by elements $\left[t_{1}\right] \cdot \ldots \cdot\left[t_{n}\right]$, with $t_{i} \in K^{\times}$. Taking $u_{0}^{i}=\left(1-t_{i}\right)^{-1}, u_{1}^{i}=1-u_{0}^{i}$, gives this generator in the desired form.

If $p \geq n \geq 0$, the universal property of $f_{n} E \rightarrow E$ gives us the isomorphism for $U \in \mathbf{S m} / k$

$$
\operatorname{Hom}_{\mathcal{S H}_{S^{1}}(k)}\left(\Sigma_{s}^{\infty} \Sigma_{\mathbb{G}_{m}}^{p} U_{+}, E\right) \cong \operatorname{Hom}_{\mathcal{S H}_{S^{1}}(k)}\left(\Sigma_{s}^{\infty} \Sigma_{\mathbb{G}_{m}}^{p} U_{+}, f_{n} E\right),
$$

since $\Sigma_{s}^{\infty} \Sigma_{\mathbb{G}_{m}}^{p} U_{+}$is in $\Sigma_{T}^{p} \mathcal{S} \mathcal{H}_{S^{1}}(k)$ for $U \in \mathbf{S m} / k$. As these groups of morphisms define the presheaves whose respective sheaves are $\Pi_{0, p} E(F)$ and $\Pi_{0, p} f_{n} E$, the $\operatorname{map} \Pi_{0, p} f_{n} E \rightarrow \Pi_{0, p} E$ is an isomorphism, hence $F_{\text {Tate }}^{n} \Pi_{0, p} E=\Pi_{0, p} E$.

Remark 7.12. The reader may object that the collection of transfer maps used to define $F_{M W^{T r}}^{n} \Pi_{0, p} E(F)$ is rather artificial. However, the fact that the general transfer maps mentioned in remark 7.5 respect the filtration $F_{\text {Tate }}^{*} \pi_{m} E$, together with theorem 7.11, shows that, if we were to allow arbitrary transfer maps in our definition of $F_{M W^{T r}}^{n} \Pi_{0, p} E(F)$, we would arrive at the same subgroup of $\Pi_{0, m} E(F)$.

Our main result for a $T$-spectrum, theorem 3 , follows easily from theorem 7.11:

Proof of theorem 3. Using lemma 2.2, we reduce to the case $p=0$. Essentially the same argument as used at the end of the proof of theorem 7.11 proves the part of theorem 3 for $n \leq 0$. 
If $n>0$, then for $b \geq 0$, we have

$$
\begin{aligned}
& \pi_{a, b} \mathcal{E} \cong \pi_{a, b} \Omega_{T}^{\infty} \mathcal{E} \\
& \pi_{a, b} f_{n} \mathcal{E} \cong \pi_{a, b} \Omega_{T}^{\infty} f_{n} \mathcal{E} \cong \pi_{a, b} f_{n} \Omega_{T}^{\infty} \mathcal{E}
\end{aligned}
$$

Thus, in case $n>0$, theorem 3 for $\mathcal{E}$ is equivalent to theorem 7.11 for $\Omega_{T}^{\infty} \mathcal{E}$, completing the proof.

Finally, we can prove our main result for the motivic sphere spectrum, theorem 1. Let $\mathcal{E}=\Sigma_{\mathbb{G}_{m}}^{q} \mathbb{S}_{k}$. Then Morel's isomorphism (7.4) and lemma 2.2 give

$$
\Pi_{a, b} \Omega_{T}^{\infty} \mathcal{E}= \begin{cases}\underline{K}_{q-b}^{M W} & \text { for } a=0, b \geq 0 \\ 0 & \text { for } a<0, b \geq 0 .\end{cases}
$$

Theorem 7.13. Let $k$ be a perfect field of characteristic $\neq 2$.

1. For all $n>p \geq 0, q \in \mathbb{Z}$, and all perfect field extensions $F$ of $k$, we have

$$
F_{\text {Tate }}^{n} \Pi_{0, p} \Omega_{T}^{\infty} \Sigma_{\mathbb{G}_{m}}^{q} \mathbb{S}_{k}(F)=K_{q-p}^{M W}(F) I(F)^{N} \subset K_{q-p}^{M W}(F),
$$

where $N=N(n-p, n-q):=\max (0, \min (n-p, n-q))$. In particular,

$$
F_{\text {Tate }}^{n} \pi_{0,0} \mathbb{S}_{k}(F)=I(F)^{n} \subset \mathrm{GW}(F) .
$$

2. For $n \leq p$, we have the identity of sheaves $F_{\text {Tate }}^{n} \Pi_{0, p} \Omega_{T}^{\infty} \Sigma_{\mathbb{G}_{m}}^{q} \mathbb{S}_{k}=\underline{K}_{q-p}^{M W}$.

3. In case $k$ has characteristic zero, we have the identity of sheaves

$$
F_{\text {Tate }}^{n} \Pi_{0, p} \Omega_{T}^{\infty} \Sigma_{\mathbb{G}_{m}}^{q} \mathbb{S}_{k}=\underline{K}_{q-p}^{M W} \mathcal{I}^{N} \subset \underline{K}_{q-p}^{M W} .
$$

with $N$ as above.

Proof. Let $N$ be as defined in the statement of the theorem. We first note (3) follows from (1), in fact, from (1) for all fields extensions $F$ finitely generated over $k$. Indeed, $F_{\text {Tate }}^{n} \Pi_{0, p} \Omega_{T}^{\infty} \Sigma_{\mathbb{G}_{m}}^{q} \mathbb{S}_{k}$ is the image of the map

$$
\Pi_{0, p} f_{n} \Omega_{T}^{\infty} \Sigma_{\mathbb{G}_{m}}^{q} \mathbb{S}_{k} \rightarrow \Pi_{0, p} \Omega_{T}^{\infty} \Sigma_{\mathbb{G}_{m}}^{q} \mathbb{S}_{k}
$$

induced by the canonical morphism $f_{n} \Omega_{T}^{\infty} \Sigma_{\mathbb{G}_{m}}^{q} \mathbb{S}_{k} \rightarrow \Omega_{T}^{\infty} \Sigma_{\mathbb{G}_{m}}^{q} \mathbb{S}_{k}$. By results of Morel $[9$, theorem 3 and lemma 5$]$, both homotopy sheaves are strictly $\mathbb{A}^{1}$ invariant sheaves of abelian groups. But the category of strictly $\mathbb{A}^{1}$-invariant sheaves of abelian groups is abelian [9, lemma 6.2.13], hence $F_{\text {Tate }}^{n} \Pi_{0, p} \Omega_{T}^{\infty} \Sigma_{\mathbb{G}_{m}}^{q} \mathbb{S}_{k}$ is also strictly $\mathbb{A}^{1}$-invariant. It follows, e.g., from Morel's isomorphism

$$
\pi_{0} \Omega_{T}^{\infty} \Sigma_{\mathbb{G}_{m}}^{m} \mathbb{S} \cong \pi_{-m,-m} \mathbb{S} \cong \underline{K}_{m}^{M W}
$$


that the sheaves $\underline{K}_{m}^{M W}$ are strictly $\mathbb{A}^{1}$-invariant; as $\underline{K}_{q-p}^{M W} \mathcal{I}^{N}$ is the image of the map

$$
\times \eta^{M}: \underline{K}_{q-p+M}^{M W} \rightarrow \underline{K}_{q-p}^{M W},
$$

where $M=N$ if $q-p \geq 0, M=p-q+N$ if $q-p<0$, it follows that $\underline{K}_{q-p}^{M W} \mathcal{I}^{N}$ is strictly $\mathbb{A}^{1}$-invariant as well. Our assertion follows from the fact that a strictly $\mathbb{A}^{1}$-invariant sheaf $\mathcal{F}$ is zero if and only $\mathcal{F}(k(X))=0$ for all $X \in \mathbf{S m} / k$, which in turn is an easy consequence of [11, lemma 3.3.6].

Next, suppose $n-p \leq 0$. Then $N=0$ and

$$
\begin{array}{rlr}
F_{\text {Tate }}^{n} \Pi_{0, p} \Omega_{T}^{\infty} \Sigma_{\mathbb{G}_{m}}^{q} \mathbb{S}_{k} & =F_{\text {Tate }}^{n-p} \Pi_{0,0} \Omega_{\mathbb{G}_{m}}^{p} \Omega_{T}^{\infty} \Sigma_{\mathbb{G}_{m}}^{q} \mathbb{S}_{k} & \text { (lemma 2.2) } \\
& =\Pi_{0,0} \Omega_{\mathbb{G}_{m}}^{p} \Omega_{T}^{\infty} \Sigma_{\mathbb{G}_{m}}^{q} \mathbb{S}_{k} & (n-p<0) \\
& =\Pi_{0, p} \Omega_{T}^{\infty} \Sigma_{\mathbb{G}_{m}}^{q} \mathbb{S}_{k} & \text { (adjunction) } \\
& =\underline{K}_{q-p}^{M W} & \text { (Morel's theorem) }
\end{array}
$$

proving (2); we may thus assume $n-p>0$.

By (7.4), we may apply theorem 7.11, which tells us $F_{\text {Tate }}^{n} \Pi_{0, p} \Omega_{T}^{\infty} \Sigma_{\mathbb{G}_{m}}^{q} \mathbb{S}_{k}(F)$ is the subgroup of $\Pi_{0, p} \Omega_{T}^{\infty} \Sigma_{\mathbb{G}_{m}}^{q} \mathbb{S}_{k}(F)=K_{q-p}^{M W}(F)$ generated by elements of the form $\operatorname{Tr}_{F}(w)^{*}(y \cdot x)$ with

$$
\begin{aligned}
& y \in \Pi_{0, n} \Omega_{T}^{\infty} \Sigma_{\mathbb{G}_{m}}^{q} \mathbb{S}_{k}(F(w))=K_{q-n}^{M W}(F(w)) \\
& x \in K_{n-p}^{M W}(F(w)) .
\end{aligned}
$$

Suppose that $n-q<0$, so $N=0$. Then $q-n \geq 0$ and $n-p>0$, and thus the product map

$\mu_{n-p, q-n}: K_{n-p}^{M W}(F(w)) \otimes K_{q-n}^{M W}(F(w)) \rightarrow K_{q-p}^{M W}(F(w))=\Pi_{0, p} \Omega_{T}^{\infty} \Sigma_{\mathbb{G}_{m}}^{q} \mathbb{S}_{k}(F(w))$ is surjective. Since the map $\operatorname{Tr}_{F}(w)$ is an isomorphism for $w \in V_{n}(F)$, we see that

$$
F_{\text {Tate }}^{n} \Pi_{0, p} \Omega_{T}^{\infty} \Sigma_{\mathbb{G}_{m}}^{q} \mathbb{S}_{k}(F)=\Pi_{0, p} \Omega_{T}^{\infty} \Sigma_{\mathbb{G}_{m}}^{q} \mathbb{S}_{k}(F) .
$$

Suppose $n-q \geq 0$. Then

$$
\times \eta^{n-q}: K_{0}^{M W}(F(w)) \rightarrow K_{q-n}^{M W}(F(w))
$$

is surjective. If $n-p \geq n-q$, then the image of $\mu_{n-p, q-n}$ is the same as the image of the triple product

$$
K_{q-p}^{M W}(F(w)) \otimes K_{n-q}^{M W}(F(w)) \otimes K_{q-n}^{M W}(F(w)) \rightarrow K_{q-p}^{M W}(F(w)) ;
$$


as the image of

$$
\mu_{n-q, q-n}: K_{n-q}^{M W}(F(w)) \otimes K_{q-n}^{M W}(F(w)) \rightarrow K_{0}^{M W}(F(w))
$$

is $I(F(w))^{n-q}$, we see that the image of $\mu_{n-p, q-n}$ is $K_{q-p}^{M W}(F(w)) I(F(w))^{n-q}$ and thus

$$
F_{\text {Tate }}^{n} \Pi_{0, p} \Omega_{T}^{\infty} \Sigma_{\mathbb{G}_{m}}^{q} \mathbb{S}_{k}(F)=\left[\Pi_{0, p} \Omega_{T}^{\infty} \Sigma_{\mathbb{G}_{m}}^{q} \mathbb{S}_{k} \mathcal{I}^{N}\right]^{T r}(F) .
$$

Similarly, if $n-q \geq n-p$, then the image of $\mu_{n-p, q-n}$ is the same as the image of the triple product

$$
K_{q-p}^{M W}(F(w)) \otimes K_{n-p}^{M W}(F(w)) \otimes K_{p-n}^{M W}(F(w)) \rightarrow K_{q-p}^{M W}(F(w))
$$

which is $K_{q-p}^{M W}(F(w)) I(F(w))^{n-p}$. Thus

$$
F_{\text {Tate }}^{n} \Pi_{0, p} \Omega_{T}^{\infty} \Sigma_{\mathbb{G}_{m}}^{q} \mathbb{S}_{k}(F)=\left[\Pi_{0, p} \Omega_{T}^{\infty} \Sigma_{\mathbb{G}_{m}}^{q} \mathbb{S}_{k} \mathcal{I}^{N}\right]^{T r}(F)
$$

in this case as well.

Thus, to complete the proof, it suffices to show that, for $w$ a closed point of $V_{n F}$, and $N \geq 0$ an integer, we have

$$
\operatorname{Tr}_{F}(w)^{*}\left(K_{q-p}^{M W}(F(w)) I(F(w))^{N}\right) \subset K_{q-p}^{M W}(F) I(F)^{N} .
$$

First suppose that $q-p \geq 0$. Take a closed point $w \in V_{n F}$ and elements $x_{1}, \ldots, x_{N} \in F(w)^{\times}, y \in K_{q-p}^{M W}(F(w))$. We have

$$
\begin{aligned}
\operatorname{Tr}_{F}(w)^{*}\left(y \cdot\left[x_{1}\right] \eta \cdot \ldots \cdot\left[x_{N}\right] \eta\right) & =\operatorname{Tr}_{F}(w)^{*}\left(y \cdot\left[x_{1}\right] \cdot \ldots \cdot\left[x_{N}\right] \eta^{N}\right) \\
& =\operatorname{Tr}_{F}(w)^{*}\left(y \cdot\left[x_{1}\right] \cdot \ldots \cdot\left[x_{N}\right]\right) \cdot \eta^{N} .
\end{aligned}
$$

where we use remark 7.10 in the last line. Since $q-p \geq 0, K_{q-p}^{M W}(F) I(F)^{N}$ is the image in $K_{q-p}^{M W}(F)$ of the map

$$
-\times \eta^{N}: K_{q-p+N}^{M W}(F) \rightarrow K_{q-p}^{M W}(F),
$$

which verifies (7.5).

In case $q-p<0$, write $y=y_{0} \eta^{p-q}$, with $y_{0} \in K_{0}^{M W}(F(w))$. As above, we have

$$
\operatorname{Tr}_{F}(w)^{*}\left(y \cdot\left[x_{1}\right] \eta \cdot \ldots \cdot\left[x_{N}\right] \eta\right)=\operatorname{Tr}_{F}(w)^{*}\left(y_{0} \cdot\left[x_{1}\right] \cdot \ldots \cdot\left[x_{N}\right]\right) \cdot \eta^{p-q+N},
$$

which is in $\eta^{p-q} \cdot\left[K_{N}^{M W}(F) \eta^{N}\right]=K_{q-p}^{M W}(F) I(F)^{N}$, as desired.

Theorem 7.13 yields the main result for the $S^{1}$-spectra $\Sigma_{s}^{\infty} \mathbb{G}_{m}^{\wedge q}$ by using the $S^{1}$-stable consequences of Morel's unstable computations, theorem 7.7. 
Corollary 7.14. Let $k$ be a perfect field of characteristic $\neq 2$.

1. For all $n>p \geq 0, q \geq 1$, and all perfect field extensions $F$ of $k$, we have

$$
F_{\text {Tate }}^{n} \Pi_{0, p} \Sigma_{s}^{\infty} \mathbb{G}_{m}^{\wedge q}(F)=K_{q-p}^{M W}(F) I(F)^{N(n-p, n-q)} \subset K_{q-p}^{M W}(F),
$$

with $N(n-p, n-q)$ as in theorem 7.13.

2. For $n \leq p$, we have $F_{\text {Tate }}^{n} \Pi_{0, p} \Sigma_{s}^{\infty} \mathbb{G}_{m}^{\wedge q}=\Pi_{0, p} \Sigma_{s}^{\infty} \mathbb{G}_{m}^{\wedge q}$.

3. If char $k=0$, we have the identity of sheaves

$$
F_{\text {Tate }}^{n} \Pi_{0, p} \Sigma_{s}^{\infty} \mathbb{G}_{m}^{\wedge q}=\underline{K}_{q-p}^{M W} \mathcal{I}^{N(n-p, n-q)} \subset \underline{K}_{q-p}^{M W} .
$$

Proof. As in the proof of theorem 7.13, it suffices to prove (1).

The main point is that Morel's unstable computations show that the $\mathbb{G}_{m^{-}}$ stabilization map

$$
\begin{aligned}
\operatorname{Hom}_{\mathcal{S H}_{S^{1}}(k)}\left(\Sigma_{s}^{m} \Sigma_{s}^{\infty} \mathbb{G}_{m}^{\wedge p}\right. & \left.\wedge \operatorname{Spec} F_{+}, \Sigma_{s}^{\infty} \mathbb{G}_{m}^{\wedge q}\right) \\
& \rightarrow \operatorname{Hom}_{\mathcal{S} \mathcal{H}_{S^{1}}(k)}\left(\Sigma_{s}^{m} \Sigma_{s}^{\infty} \mathbb{G}_{m}^{\wedge p+1} \wedge \operatorname{Spec} F_{+}, \Sigma_{s}^{\infty} \mathbb{G}_{m}^{\wedge q+1}\right)
\end{aligned}
$$

is an isomorphism for all $m \leq 0, p \geq 0$ and $q \geq 1$.

Let $E(p, q)=\Omega_{\mathbb{G}_{m}}^{p} \Sigma_{s}^{\infty} \mathbb{G}_{m}^{\wedge q}$, and let

$$
E(q-p)=\Omega_{T}^{\infty} \Sigma_{\mathbb{G}_{m}}^{-p} \Sigma_{T}^{\infty} \mathbb{G}_{m}^{\wedge q}=\Omega_{T}^{\infty} \Sigma_{\mathbb{G}_{m}}^{q-p} \mathbb{S}_{k} .
$$

Then

$$
\pi_{a} E(p, q)=\Pi_{a, p} \Sigma_{s}^{\infty} \mathbb{G}_{m}^{\wedge q} .
$$

Thus $\Pi_{a, *} E(p, q)=0$ for $m<0$ and so we may apply proposition 4.3 to give generators of the form $\xi_{w} \circ \Sigma_{s}^{\infty} Q_{F}(w)$ for

$$
F_{\text {Tate }}^{n-p} \Pi_{0,0} \Omega_{\mathbb{G}_{m}}^{p} \Sigma_{s}^{\infty} \mathbb{G}_{m}^{\wedge q}(F)=F_{\text {Tate }}^{n} \Pi_{0, p} \Sigma_{s}^{\infty} \mathbb{G}_{m}^{\wedge q}(F) .
$$

But $\xi_{w}$ is in

$$
\pi_{-n+p} \Omega_{T}^{n-p} E(p, q)(w)=\pi_{0, n-p} E(p, q)(w) .
$$

Similarly, we have generators $\xi_{w}^{\prime} \circ \Sigma_{s}^{\infty} Q_{F}(w)$ for $F_{\text {Tate }}^{n-p} \pi_{0} E(p-q)(F)$, with

$$
\xi_{w}^{\prime} \in \pi_{0, n-p} E(p-q)(w) .
$$

But the stabilization map

$$
\pi_{0, n-p} E(p, q)(w) \rightarrow \pi_{0, n-p} E(p+1, q+1)(w)
$$


is an isomorphism, and hence we have an isomorphism from the generators for $F_{\text {Tate }}^{n-p} \pi_{0} E(p, q)(F)$ to the generators for

$$
F_{\text {Tate }}^{n-p} \pi_{0} E(q-p)(F)=\underset{m}{\lim _{\text {Tate }}} F_{0}^{n-p} E(p+m, q+m)(F) .
$$

As the map

$$
\pi_{0} E(p, q)(F) \rightarrow \pi_{0} E(q-p)(F)=K_{q-p}^{M W}(F)
$$

is an isomorphism, it follows that the surjection

$$
F_{\text {Tate }}^{n-p} \pi_{0} E(q-p)(F) \rightarrow F_{\text {Tate }}^{n-p} \pi_{0} E(q-p) .
$$

is an isomorphism as well. By theorem 7.13, we have

$$
F_{\text {Tate }}^{n-p} \pi_{0} E(q-p)=K_{q-p}^{M W}(F) I(F)^{N} \subset K_{q-p}^{M W}(F),
$$

completing the proof.

Theorem 7.13 also gives us the $T$-stable version

Corollary 7.15. Let $k$ be a perfect field of characteristic $\neq 2$. For $n, p, q \in \mathbb{Z}$, and $F$ a perfect field extensions of $k$, we have

$$
F_{\text {Tate }}^{n} \pi_{p, p} \Sigma_{\mathbb{G}_{m}}^{q} \mathbb{S}_{k}(F)=K_{q-p}^{M W}(F) I(F)^{N(n-p, n-q)} \subset K_{q-p}^{M W}(F)
$$

For $n \leq p$, we have $F_{\text {Tate }}^{n} \pi_{p, p} \Sigma_{\mathbb{G}_{m}}^{q} \mathbb{S}_{k}=\underline{K}_{q-p}^{M W}$. If char $k=0$, we have

$$
F_{\text {Tate }}^{n} \pi_{p, p} \Sigma_{\mathbb{G}_{m}}^{q} \mathbb{S}_{k}=\underline{K}_{q-p}^{M W} \mathcal{I}^{N(n-p, n-q)} \subset \underline{K}_{q-p}^{M W} .
$$

Proof. Using lemma 2.2 and lemma 7.8 as in the proof of theorem 7.11 we have

$$
F_{\text {Tate }}^{n} \pi_{p, p} \Sigma_{\mathbb{G}_{m}}^{q} \mathbb{S}_{k}=F_{\text {Tate }}^{n-p+r} \pi_{r, r} \Sigma_{\mathbb{G}_{m}}^{q-p+r} \mathbb{S}_{k}
$$

for all integers $r$. As our assertion is also stable under this shift operation, we may assume that $p, q \geq 0$. We note that $\mathbb{S}_{k}$ is in $\mathcal{S H}^{e f f}(k)$, hence so are all $\Sigma_{\mathbb{G}_{m}}^{q} \mathbb{S}_{k}$ for $q \geq 0$, and thus

$$
F_{\text {Tate }}^{n} \pi_{p, p} \Sigma_{\mathbb{G}_{m}}^{q} \mathbb{S}_{k}=\pi_{p, p} \Sigma_{\mathbb{G}_{m}}^{q} \mathbb{S}_{k}
$$

for $n<0, p, q \geq 0$. The truncation functors $f_{n}, n \geq 0$, on $\mathcal{S H}(k)$ and $\mathcal{S H}_{S^{1}}(k)$ commute with $\Omega_{T}^{\infty}$, and $\pi_{a, p} \Omega_{T}^{\infty} \mathcal{E}=\pi_{a, p} \mathcal{E}$ for $\mathcal{E} \in \mathcal{S H}(k), p \geq 0$. This reduces us to computing computing $F_{\text {Tate }}^{n} \pi_{p, p} \Omega_{T}^{\infty} \Sigma_{\mathbb{G}_{m}}^{q} \mathbb{S}_{k}$ for $n, p, q \geq 0$, which is theorem 7.13. 


\section{Epilog: Convergence QUeStions}

Voevodsky has stated a conjecture [14, conjecture 13] that would imply that for $\mathcal{E}=\Sigma_{T}^{\infty} X_{+}, X \in \mathbf{S m} / k$, the Tate Postnikov tower is convergent in the following sense: for all $a, b, n \in \mathbb{Z}$, one has

$$
\cap_{m} F_{\text {Tate }}^{m} \pi_{a, b} f_{n} \mathcal{E}=0 .
$$

Our computation of $F_{\text {Tate }}^{n} \pi_{p, p} \Sigma_{T}^{\infty} \mathbb{G}_{m}^{\wedge q}$ gives some evidence for this convergence conjecture.

Proposition 8.1. Let $k$ be a perfect field with char $k \neq 2$. For all $p, q \geq 0$, and all perfect field extensions $F$ of $k$, we have

$$
\cap_{n} F_{\text {Tate }}^{n} \pi_{p, p} \Sigma_{T}^{\infty} \mathbb{G}_{m}^{\wedge q}(F)=0 .
$$

Proof. In light of theorem 7.13, the assertion is that the $I(F)$-adic filtration on $K_{q-p}^{M W}(F)$ is separated. By [10, théorème 5.3], for $m \geq 0, K_{m}^{M W}(F)$ fits into a cartesian square of $\mathrm{GW}(F)$-modules

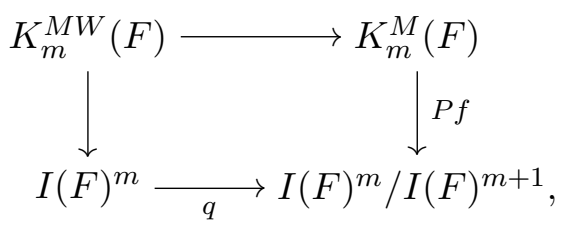

where $K_{m}^{M}(F)$ is the Milnor $K$-group, $q$ is the quotient map and $P f$ is the map sending a symbol $\left\{u_{1}, \ldots, u_{m}\right\}$ to the class of the Pfister form $\left\langle<u_{1}, \ldots, u_{m}\right\rangle>$ $\bmod I(F)^{m+1}$. For $m<0, K_{m}^{M W}(F)$ is isomorphic to the Witt group of $F$, $W(F)$, that is, the quotient of $\mathrm{GW}(k)$ by the ideal generated by the hyperbolic form $x^{2}-y^{2}$. Also, the map $\mathrm{GW}(F) \rightarrow W(F)$ gives an isomorphism of $I(F)^{r}$ with its image in $W(F)$ for all $r \geq 1$. Thus

$$
K_{m}^{M W}(F) I(F)^{n}= \begin{cases}I(F)^{n} \subset W(F) & \text { for } m<0, n \geq 0 \\ I(F)^{n+m} \subset \mathrm{GW}(F) & \text { for } m \geq 0, n \geq 1\end{cases}
$$

The fact that $\cap_{n} I(F)^{n}=0$ in $W(F)$ or equivalently in $\mathrm{GW}(F)$ is a theorem of Arason and Pfister [1].

Remarks 8.2. 1. The proof in [10] that $K_{m}^{M W}(F)$ fits into a cartesian square as above relies the Milnor conjecture. 
2. Voevodsky's conjecture [loc. cit.] asserts the convergence for a wider class of objects in $\mathcal{S H}(k)$ than just the $T$-suspension spectra of smooth $k$-schemes. The selected class is the triangulated category generated by $\Sigma_{T}^{n} \Sigma_{T}^{\infty} X_{+}, X \in \mathbf{S m} / k$, $n \in \mathbb{Z}$ and the taking of direct summands. However, as pointed out to me by Igor Kriz, the convergence fails for this larger class of objects. In fact, take $\mathcal{E}$ to be the Moore spectrum $\mathbb{S}_{k} / \ell$ for some prime $\ell \neq 2$. Since $\Pi_{a, q} \mathbb{S}_{k}=0$ for $a<0$, proposition 3.2 shows that $\Pi_{a, q} f_{n} \mathbb{S}_{k}=0$ for $a<0$, and thus we have the right exact sequence for all $n \geq 0$

$$
\pi_{0,0} f_{n} \mathbb{S}_{k} \stackrel{\times \ell}{\longrightarrow} \pi_{0,0} f_{n} \mathbb{S}_{k} \rightarrow \pi_{0,0} f_{n} \mathcal{E} \rightarrow 0 .
$$

In particular, we have

$$
F_{\text {Tate }}^{n} \pi_{0,0} \mathcal{E}(k)=i m\left(F_{\text {Tate }}^{n} \pi_{0,0} \mathbb{S}_{k}(k) \rightarrow \pi_{0,0} \mathbb{S}_{k}(k) / \ell\right)=i m\left(I(k)^{n} \rightarrow \mathrm{GW}(k) / \ell\right) .
$$

Take $k=\mathbb{R}$. Then $G W(\mathbb{R})=\mathbb{Z} \oplus \mathbb{Z}$, with virtual rank and virtual index giving the two factors. The augmentation ideal $I(\mathbb{R})$ is thus isomorphic to $\mathbb{Z}$ via the index and it is not hard to see that $I(\mathbb{R})^{n}=\left(2^{n-1}\right) \subset \mathbb{Z}=I(\mathbb{R})$. Thus $\pi_{0,0} \mathcal{E}=\mathbb{Z} / \ell \oplus \mathbb{Z} / \ell$ and the filtration $F_{\text {Tate }}^{n} \pi_{0,0} \mathcal{E}$ is constant, equal to $\mathbb{Z} / \ell=I(\mathbb{R}) / \ell$, and is therefore not separated.

The convergence property is thus not a "triangulated" one in general, and therefore seems to be quite subtle. However, if the $I$-adic filtration on $\mathrm{GW}(F)$ is finite (possibly of varying length depending on $F$ ) for all finitely generated $F$ over $k$, then our computations (at least in characteristic zero) show that the filtration $F_{\text {Tate }}^{*} \pi_{p, p} \Sigma_{T}^{\infty} \mathbb{G}_{m}^{\wedge q}$ is at least locally finite, and thus has better triangulated properties; in particular, for $\ell \neq 2$,

$$
\pi_{0,0}\left(\mathbb{S}_{k} / \ell\right)=\mathbb{Z} / \ell, F_{\text {Tate }}^{n} \pi_{0,0}\left(\mathbb{S}_{k} / \ell\right)=0 \text { for } n>0,
$$

as the augmentation ideal in $\mathrm{GW}(F)$ is purely two-primary torsion, and thus $\mathcal{I} \pi_{0,0} \mathbb{S}_{k} / \ell=0$. One can therefore ask if Voevodsky's convergence conjecture is true if one assumes the finiteness of the $I(F)$-adic filtration on $\mathrm{GW}(F)$ for all finitely generated fields $F$ over $k$.

\section{REFERENCES}

[1] Arason, J. K. and Pfister, A., Beweis des Krullschen Durchschnittsatzes für den Wittring. Invent. Math. 12 (1971), 173-176.

[2] Bousfield, A. K. and Kan, D. M.Homotopy limits, completions and localizations. Lecture Notes in Mathematics, 304. Springer-Verlag, Berlin-New York, 1972. 
[3] Goerss, P. G.; Jardine, J. F., Localization theories for simplicial presheaves. Canad. J. Math. 50 (1998), no. 5, 1048-1089.

[4] Jardine, J. F., Simplicial presheaves, J. Pure Appl. Algebra 47 (1987), no. 1, 35-87

[5] Jardine, J.F., Motivic symmetric spectra, Doc. Math. 5 (2000), 445-553.

[6] Levine, M., The homotopy coniveau tower. J Topology 1 (2008) 217-267.

[7] Levine, M., Chow's moving lemma in $\mathbb{A}^{1}$-homotopy theory. $K$-theory 37 (1-2) (2006) 129 209.

[8] Morel, F., $\mathbb{A}^{1}$-Algebraic topology over a field, preprint 19.11.2006. http://www.mathematik.uni-muenchen.de/ morel/A1homotopy.pdf

[9] Morel, F., The stable $\mathbb{A}^{1}$-connectivity theorems, $K$-theory, 2005, 35, pp 1-68.

[10] Morel, F. Sur les puissances de l'idéal fondamental de l'anneau de Witt. Comment. Math. Helv. 79 (2004), no. 4, 689-703.

[11] Morel, F., An introduction to $\mathbb{A}^{1}$-homotopy theory. Contemporary developments in algebraic K-theory 357-441, ICTP Lect. Notes, XV, Abdus Salam Int. Cent. Theoret. Phys., Trieste, 2004.

[12] Morel, F. and Voevodsky, V., $\mathbb{A}^{1}$-homotopy theory of schemes, Inst. Hautes Études Sci. Publ. Math. 90 (1999), 45-143.

[13] Neeman, A, Triangulated categories. Annals of Mathematics Studies, 148. Princeton University Press, Princeton, NJ, 2001.

[14] Voevodsky, V., Open problems in the motivic stable homotopy theory. I. Motives, polylogarithms and Hodge theory, Part I (Irvine, CA, 1998), 3-34, Int. Press Lect. Ser., 3, I, Int. Press, Somerville, MA, 2002.

[15] Voevodsky, V. A possible new approach to the motivic spectral sequence for algebraic K-theory, Recent progress in homotopy theory (Baltimore, MD, 2000) 371-379, Contemp. Math., 293 (Amer. Math. Soc., Providence, RI, 2002).

\section{Marc Levine}

Universität Duisburg-Essen

Fakultät Mathematik, Campus Essen

45117 Essen, Germany

E-mail: marc.levine@uni-due.de 